\title{
Enhancement of Heat Transfer in the Bundles of Transversely-Finned Tubes
}

\author{
Pis'mennyi, E.N., Terekh, A.M. and Razumovskiy, V.G. \\ National Technical University of Ukraine "Kyiv Polytechnic Institute"
}

Ukraine

\section{Introduction}

The problem of improving heat transfer surfaces made in the form of the bundles of transversely finned tubes remains very pressing. Studying the works, devoted to this problem, and a number of patents, including patents of the USA, Great Britain, Germany, and Japan, revealed that the ways of increasing thermoaerodynamic efficiency of the transversely finned surfaces mainly involve a search for the most rational types of finning and arrangement of the finned tube bundles.

A great many works on developing intensified surfaces are associated with setting up conditions for the breakdown of thickened boundary layers on relatively high fins and for the organization of a developed vortex flow, if possible, over the entire surface. Such conditions are attained by corrugation of the transverse fins (Tolubinskiy \& Lyogkiy, 1964), their perforation (Migai et al., 1992; Eckels \& Rabas, 1985), cutting into short sections with ends bent to the opposite sides (Taranyan et al., 1972; Sparrow \& Myrum, 1985) and with the use of the so-called segment finning.

Analyzing these developmental works and related investigations allows us to note the following. The fin corrugation leads to a noticeable augmentation of heat transfer, but the heat enhancement is accompanied in this case by a still more noticeable increase of the aerodynamic drag: by data (Tolubinskiy \& Lyogkiy, 1964), the replacement of smooth fins on a single finned cylinder by corrugated fins at $\operatorname{Re}=10^{4}$ enhances heat transfer by $12-15 \%$ with a drag increase by $65-70 \%$. This circumstance, in conjunction with a difficult manufacturability of the tubes with corrugated fins, renders their wide use problematic.

The literature offers ample coverage of the results for thermoaerodynamic characteristics of bundles of tubes with cut fins (Taranyan et al., 1972; Kuntysh \& Iokhvedov, 1968; Antufiev \& Gusyev, 1968; Iokhvedov et al., 1975; Antufiev, 1965) (Fig. 1). Such heat transfer surfaces are fabricated from the tubes with a typical helical finning by cutting the fins into short sections by a thin mill along the generatrix of a carrying cylinder or along a helical line at an angle of $45^{\circ}$. Cutting does not practically diminish the fin surface and, according to data of the above-mentioned works, enhances heat transfer by $12-36 \%$ depending on the fin parameters and the method of cutting fins. The effect of flow turbulization, produced in this case, is the more appreciable, the higher are the cut fins. However, in all cases an increase in aerodynamic drag markedly outstrips an increase in heat transfer which on the whole noticeably reduces the total effect of heat transfer enhancement. Besides, the production of tubes with cut fins requires additional technological operations, which, in conjunction with 
a high susceptibility to contamination of the heat transfer surfaces from such tubes and complexity of their cleaning, substantially limited their application.
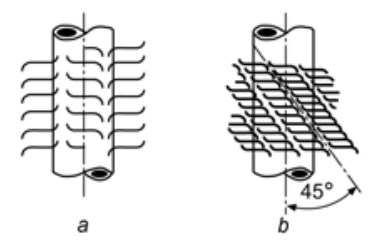

Fig. 1. Tubes with cut fins (Taranyan et al., 1972; Kuntysh \& Iokhvedov, 1968; Iokhvedov et al., 1975)

More technologically advantageous, with similar characteristics of thermoaerodynamic efficiency, is a solution involving the application of flow-agitating notches on the ends of rolled-on fins (Kokorev et al., 1978; Kuntysh \& Piir, 1991; Kuntysh, 1993), owing to which it found use in manufacturing heat transfer surfaces from aluminum tubes for some air cooling devices. However, this situation, apart from the limited range of application as to temperature conditions, retained essential operational drawbacks of the cut fins with bent edges, viz. an increased susceptibility to contamination and complexity of cleaning the interfin gaps, aggravated by their considerable blocking by the deformed fin edges.

Fairly well-known is the type of finning referred to in the literature as segment finning (Fig. 2). Judging from advertizing materials and from data (Weierman, 1976), such finning can produce an appreciable effect even in the case of some loss by heat transfer surface in comparison with continuous fins with the same height, thickness and pitch. Heat transfer is enhanced here not only because of a decrease in the boundary layer thickness as a result of a small width of an individual "segment" and the turbulization of flow on its separation from sharp edges of the fin but also because each individual element of the segment fin is, in essence, a straight rectangular fin with efficiency higher than of a disk fin. Estimates according to data (Weierman, 1976) manifest that replacing a typical helical finning by segment finning, with other conditions being equal, can decrease the number of tubes in a bundle by $18-20 \%$. However, reviewing the structures of present-day heat exchangers, including power devices, indicates that, regardless of the above-stated assets that are combined with easier manufacturability (the segment finning is formed by welding a preliminarily notched steel strip to the tube by high-frequency currents), the considered type of the intensified heat transfer surface has not found wide application as yet. This fact is to some extent linked with a scanty investigation of thermoaerodynamic characteristics of the surfaces from tubes with segment finning. Insufficiently reliable, by our data, are design equations forming the basis for estimating thermoaerodynamic characteristics of the bundles of such tubes.

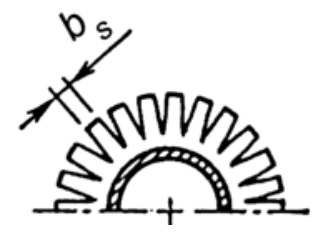

Fig. 2. Segmented fins (Weierman, 1976) 
The enhancement method, described in (Fiebig et al., 1990), also involves perforation of a thin fin. Its essence lies in the formation, on a rectangular fin behind the carrying cylinder, of two delta wings representing bent parts of the perforated fin. The wings, inclined toward the incident flow, generate longitudinal vortices enhancing transfer in the near-wall region, which, with reference to actual heat exchangers, in the authors' opinion, can increase heat transfer by $20 \%$ and decrease operational expenditure by $10 \%$. These estimates, relying on the exploration of experimental data for a single finned cylinder to a multirow heat exchanger, are too optimistic, considering the variation in the flow turbulence over the depth of a finned bundle.

Study (Kuntysh \& Kuznetsov, 1992) attributes the improvement of mass and dimensional characteristics of the surfaces, made from circular tubes with a helical rolled-on finning, to the removal of a finned part lying in the wake region behind the carrying cylinder, where the heat transfer rate on the whole, as is well known, is relatively low. For the removal of the finned part to be possibly more adaptable to manufacture, the authors suggest that fins should be cut off on the chord along the plane parallel to the tube midsection (Fig. 3). According to data (Kuntysh \& Kuznetsov, 1992), the heat transfer coefficients, related to a total surface of the tubes with a finning cutoff in the indicated fashion throughout height $h$, increase in comparison with the case of typical finned tubes by 1.23 times at $\operatorname{Re}=3 \cdot 10^{3}$ and by 1.3 times at $\operatorname{Re}=2.5 \cdot 10^{4}$. Here, the aerodynamic drag is practically unchangeable. However, due to the decrease in the area of the heat transfer surface, a total heat extraction diminishes by $13 \%$ and $23 \%$, respectively. Nonetheless, the authors assert that, with other conditions being equal, up to $28 \%$ of the metal consumed for the finning fabrication can thus be saved. Overall, the above-considered way of perfecting transversely finned surfaces cannot be recognized as rational for obvious reasons.

Yet another trend for improving thermoaerodynamic characteristics of the tubular transversely finned surfaces that involves a change of their geometry is a search for new types of the arrangement of finned tube bundles. Main ideas of such developmental works have been mostly borrowed from studies (Yevenko \& Anisin, 1976; Lokshin et al., 1982; Migai \& Firsova, 1986) conducted with smooth tube bundles. Among them are:

- a so-called crossed or lattice arrangement of the tubes;

- $\quad$ arrangements intermediate between purely in-line and purely staggered that can be produced by changing the angle between the axes of longitudinal rows of ordinary inline tube bundles and the velocity vector of the incident flow;

- zigzag arrangements formed by an alternating longitudinal displacement of the tubes in transverse rows of staggered bundles (Fig. 4); and

- $\quad$ bundles with an unequal number of tubes in transverse row.

A significant volume of investigations in these directions has been conducted in works (Kuntysh \& Kuznetsov, 1992; Kuntysh et al., 1991; Kuntysh \& Stenin, 1993; Stenin, 1994; Kuntysh et al., 1990).

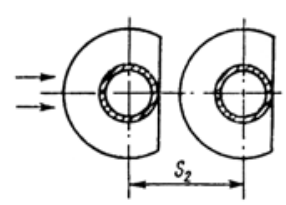

Fig. 3. Tubes with finning cut off in the rear part 
When characterizing the results for thermoaerodynamic characteristics of crossed bundles it should be noted that, in conformity with data (Kuntysh \& Kuznetsov, 1992), such arrangements do not enhance heat transfer in comparison with an ordinary layout of finned tubes, although the drag decreases somewhat, and should in all probability be regarded as inexpedient.

Studying the characteristics of intermediate in-line - staggered arrangements (Kuntysh \& Stenin, 1993; Stenin, 1994) revealed the effect of heat transfer enhancement reaching $5 \%$ relative to the data for the original purely staggered bundle with a dense distribution of tubes, which is comparable with an error of the experiments of this kind. An appreciably greater effect can be attained, as study (Pis'mennyi, 1991) showed, using normal staggered arrangements with optimal pitch relationships.

The use of zigzag arrangements can be justified to some extent primarily because the frontal width of a bundle can be diminished (Fig. 4). Discrepancy of the data (Kuntysh \& Kuznetsov, 1992) allows us to assume that there is no noticeable effect of heat transfer enhancement when the tubes in transverse rows of staggered bundles are displaced. The matter is that, in the above-mentioned study, in experiments with zigzag tube bundles with the fin factor $\psi=12.05$ and with the drag equal to that of original ordinary staggered bundles heat transfer increased by $8-17 \%$ and experiments with zigzag tube bundles with the fin factor $\psi=17.5$ indicated a decrease in the surface-average heat transfer. It is very doubtful that the recorded relatively insignificant variation in the parameters can lead to a substantial change in transfer in the bundles of transversely finned tubes. Obviously, the above effects are linked with methodical errors of the experiments.

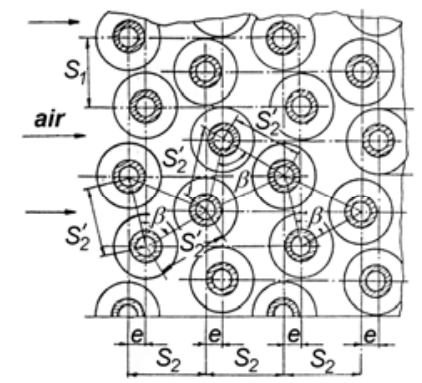

Fig. 4. Zigzag tube arrangement (Kuntysh \& Kuznetsov, 1992)

Some studies (Kuntysh \& Fedotova, 1983; Samie \& Sparrow, 1986; Khavin, 1989) considered the possibility of enhancing heat transfer by inclining the finned tubes with respect to the direction of the incident flow. In this case, an additional turbulization of the flow occurs as a result of its separation from the inlet edges of fins whose planes have a positive attack angle. Experiments, performed in the region of Reynolds numbers $\operatorname{Re}=$ $5 \cdot 10^{3}-5 \cdot 10^{4}$ with a single finned tube (Samie \& Sparrow, 1986) and with staggered tube bundles (Kuntysh \& Fedotova, 1983), showed an increase the surface in average heat transfer coefficients by $20-30 \%$ with an increase in the inclination angle by up to 40 . Here, the most intense rise in $a$ is observed for the inclination angles raging from 0 to $30^{\circ}$, which, with a relatively monotonic increase in the aerodynamic drag, allows a selection of an optimal inclination angle of the tubes. 
A certain reserve for increasing thermoaerodynamic efficiency of transversely finned tubes resides in converting to a noncircular shape of the cross section of the fin-carrying tube. Under definite conditions, specifically, with stringent limitations on the aerodynamic drag of a heat exchanger, it is reasonable to use shaped (generally plane-oval or elliptical) tubes in lieu of circular carrying tubes (Antufiev, 1966; Berman, 1965; Yudin \& Fedorovich, 1992; Ilgarubis et al., 1987). Geometric characteristics of such tube bundles include additional parameters among which are the relation of longitudinal and lateral dimensions of the cylinder cross section and the attack angle of the profile with respect to the direction of the incident flow (Fig. 5). As works on heat transfer and aerodynamics of smooth and finned shaped cylinders (Antufiev, 1966; Yudin \& Fedorovich, 1992; Ilgarubis et al., 1987) demonstrated, the search for optimal values of these parameters can be tied with the prospects for improving the developed surfaces.

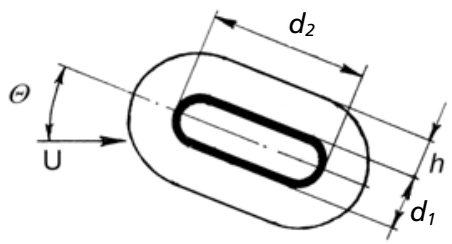

Fig. 5. Geometric characteristics of a plane-oval finned tube

In conclusion of the review part it should be noted that at the present time, in connection with a significant increase in the metal cost for large volumes of the production of the heat exchange equipment, it is considered expedient to use the ideas and designs leading to decrease in the specific amount of metal per structure only by a few percent, with other conditions being equal. Here, a good deal of attention is given to manufacturability of the developed surfaces: their elements should be fabricated with the aid of waste-free highefficient technologies (like welding, rolling on and molding).

\section{Physical substantiation of the proposed designs}

In order to determine the ways of enhancement of local heat transfer in the bundles of transversely finned tubes an experimental research of the effects of fin tube geometry, of tube type, and of in-line and staggered arrangement on the distribution of heat transfer coefficients over the fin surface was conducted in NTUU "Kiev Polytechnic Institute".

In addition, the relationships governing local heat transfer incident to gas flows across bundles of tubes with radial and helically-wound fins are of interest in calculating the temperature distributions over the heating surfaces (especially at high loads), and in determination of the real fin efficiencies $E$. The latter depend strongly upon the distribution of heat transfer coefficients $a$ over the fin surface, and are used in analytical engineering methods for converting from convection to reduced values of heat transfer coefficients.

The measurement of the distribution of $a$ over a fin involves great procedural difficulties, so relatively little was done on this problem.

Here we present the results of the studies on the local heat transfer coefficients in tubes with radial fins of different geometries, operating in various bundle arrangements. 
We worked with three tube models, described in Table 1 . The model geometries conformed to those of industrial use. In addition, we aimed to investigate the widest possible range of ratio $h / d$ which, from previous visual observations, we knew to have a large effect on the flow pattern over the fin. The tubes were assembled into staggered and in-line bundles (Table 2) and the measurements were performed in the first rows encountered by the gas flow, as well as in inner rows.

The local values of $a$ were determined under steady conditions by means of foil-type heat flux rate transducers, known as the Gardon transducers (Gardon, 1960). Pickups of this type have a fairly high sensitivity, low inertia, linear dependence of the output signal on the sensed heat flux, and are relatively simple to construct. Six to seven such pickups, with thermocouples for determining the fin-surface temperature embedded in their casings, were spaced along the mid-height of copper fins of the tubes in which the heat flux was metered. The sensing elements of the pickups were $0.05-\mathrm{mm}$ thick constantan foil disks of $6-\mathrm{mm}$ diameter. The number of pickups per tube depended on the desired radial resolution of the measurements (i.e., over the height of the fin). Variations in azimuth were obtained by turning the entire heat-metering tube about its longitudinal axis in $5^{\circ}$ to $10^{\circ}$ increments. The three types of electrically-heated heat-metering tubes used in the experiments were identical in all respects except for dimensions.

\begin{tabular}{|c|c|c|c|c|c|c|}
\hline $\begin{array}{c}\text { Type of } \\
\text { tube }\end{array}$ & $\begin{array}{c}\text { Diameter } \\
\text { of tube } \\
\text { supporting } \\
\text { the fins }\end{array}$ & $\begin{array}{c}\text { Fin } \\
\text { height } d, \\
\mathrm{~mm}\end{array}$ & $\begin{array}{c}\text { Fin pitch } \\
t, \mathrm{~mm}\end{array}$ & $\begin{array}{c}\text { Fin } \\
\text { thickness } \\
S, \mathrm{~mm}\end{array}$ & $\begin{array}{c}\text { Relative } \\
\text { fin height } \\
h / d\end{array}$ & $\begin{array}{c}\text { Surface } \\
\text { area } \\
\text { extension } \\
\text { factor } \psi\end{array}$ \\
\hline 1 & 32.0 & 30.0 & 12.7 & 5.0 & 0.932 & 10.9 \\
\hline 2 & 63.0 & 22.5 & 12.0 & 2.5 & 0.357 & 6.1 \\
\hline 3 & 83.5 & 32.5 & 12.0 & 2.5 & 0.257 & 3.8 \\
\hline
\end{tabular}

Table 1. Geometric parameters of the finned tubes

\begin{tabular}{|c|c|c|c|c|c|}
\hline $\begin{array}{c}\text { Assembly } \\
\text { number }\end{array}$ & $\begin{array}{c}\text { Tube type } \\
\text { (see Table } \\
1)\end{array}$ & Arrangement & $\begin{array}{c}\text { Relative } \\
\text { transverse } \\
\text { tube pitch } \\
\sigma_{1}\end{array}$ & $\begin{array}{c}\text { Relative } \\
\text { longitudinal } \\
\text { tube pitch } \sigma_{2}\end{array}$ & $\sigma_{1} / \sigma_{2}$ \\
\hline 1 & 1 & In-line & 3.47 & 2.97 & 1.17 \\
\hline 2 & 1 & $"$ & 3.47 & 5.31 & 0.65 \\
\hline 3 & 1 & Staggered & 3.47 & 2.56 & 1.30 \\
\hline 4 & 1 & $"$ & 3.47 & 5.31 & 0.65 \\
\hline 5 & 1 & $"$ & 4.31 & 2.65 & 1.62 \\
\hline 6 & 1 & $"$ & 4.31 & 5.31 & 0.81 \\
\hline 7 & 2 & $"$ & 3.33 & 1.30 & 2.56 \\
\hline 8 & 2 & $"$ & 3.33 & 1.76 & 1.89 \\
\hline 9 & 2 & $"$ & 4.09 & 1.30 & 3.15 \\
\hline 10 & 2 & $"$ & 4.09 & 1.76 & 2.32 \\
\hline 11 & 3 & $"$ & 3.02 & 1.30 & 2.32 \\
\hline 12 & 3 & $"$ & 3.91 & 1.30 & 3.01 \\
\hline
\end{tabular}

Table 2. Geometric parameters of the bundles of finned tubes 
The studies were performed on an air loop, described in detail in (Pis'mennyi \& Lyogkiy, 1984). The values of $a$ in the sensor locations were determined from expression

$$
\alpha_{i}=\frac{q_{i}}{t_{i}-t_{f l}}
$$

where $q_{i}$ is the flux density in the pickup site; $t_{i}$ is the fin surface temperature at this site and $t_{f l}$ is the temperature of the flowing air. The values of $q_{i}$ were determined from calibration curves $q_{i}=f\left(e_{i}\right)$, relating $q_{i}$ to the value $e_{i}$ of the electrical output of the pickup.

The measurements were performed at several fixed values of Re between $1.10^{4}$ and $5 \cdot 10^{4}$. The reference dimension was diameter $d$ of the tube supporting the fins, the reference velocity was the velocity $U$ in the narrowest net free cross section of the bundle. The physical properties were taken at flow temperature $t_{f l}$. The results were worked up in the form $a_{i} / a_{a v}=f(\varphi, P)$ at $\operatorname{Re}$ $=$ const, where $\varphi$ is the angle (in the plane of the fin) measured in the frontal (i.e., facing the air flow) generatrix of the tube, and $P=r-r_{0} / R-r_{0}$ is a dimensionless coordinate, measured along the radius of the tube that made this angle $\varphi$, the point $P=0$ being the base of the fin $\left(r=r_{0}\right)$, and the fin tip $(r=R)$ having the coordinate $P=1$. The local heat transfer coefficients $a_{i}$ were related to the surface-averaged coefficient $a_{a v}$, calculated as

$$
\alpha_{a v}=\frac{\sum_{i=1}^{N} a_{i} \Delta t_{i} H_{i}}{\Delta t_{a v} \sum_{i=1}^{N} H_{i}}
$$

where $\Delta t_{a v}$ is the surface-averaged temperature difference; $\Delta t_{i}$ is the local temperature difference; $H_{i}$ is the area of heat-transferring surface adjoining the $i$ th heat flux rate pickup, and $N$ is the number of such pickups. The values of $a_{a v}$ were also calculated from correlations for the average coefficients of heat transfer of transversely-finned tubes (Pis'mennyi, 1993). The differences in the values of $a_{i} / a_{a v}$ obtained by these two procedures were less than the experimental error, which we estimate to have been no more than $14.5 \%$. Some of the results are plotted in the figures that follow, on which the distributions of $a_{i} / a_{a v}$ are shown in the form of five zones, depending on the level of this ratio.

The measurements that shed most light on the mechanisms of the processes occurring in the space between the fins and on the effect of the finned-tube geometry on the distribution of heat transfer coefficients over the fin surface are those in the first rows of bundles with fairly large relative longitudinal tube pitches $\sigma_{2}$ (Figs. 6 and 8). At these $\sigma_{2}$, the pitches cease to affect the heat transfer. These data show that the distribution of $a$ over the fins surface is very uneven, with the highest $a$ occurring in the root zones.

The shape of these distributions fits the model of the flow over a finned tube, which we have developed previously (Pis'mennyi, 1984, 1993) on the basis of experimental data.

The distinguishing feature of that model is that it reflects the existence of intensive secondary circulatory flows near the fin roots (Fig. 9). This circulation explains all the features of the flow over finned tubes and of the distributions of $a$ over their surfaces not explained by traditional theory, namely the relatively high (and in some cases peak) $a$ near fin roots, high $a$ at the boundaries of the trailing vortical zones, etc.

The distributions of $a_{i} / a_{a v}$ plotted in Fig. 6 were obtained for a type 1 tube with relatively high fins $(h / d=0.932)$, for which the flow over the front (facing the flow or "windward") half of the tube circumference is as shown in Fig. 7a. The high values of $a_{i} / a_{a v}$ at the fin base 
result from the reattachment of secondary flows to its surface (zone $A_{2}$, Fig. 7a), while the minimum in the region of $P=0.3$ to 0.35 occurs in the zone of separation $A_{3}$ where the secondary (recirculating) and primary flow collide. The static pressures, measured in the axial plane of the windward half circumference of the fin, suggest the existence of an adverse pressure gradient, which, of course, is the necessary condition for flow separation. The local peak observed at $P=0.7$ is associated with reattachment of the flow that has separated from the sharp inlet edge of the fin which, in this case, is quite thick. The shapes of the distributions of $a_{i} / a_{a v}$ do not vary greatly as Re is varied from $2 \cdot 10^{4}$ to $5 \cdot 10^{4}$. However, the peak $a_{i} / a_{a v}$ is shifted from the root region of the fin (at $\left.\operatorname{Re}=2 \cdot 10^{4}\right)$ to the region of its front edge (at $\left.\operatorname{Re}=5 \cdot 10^{4}\right)$.

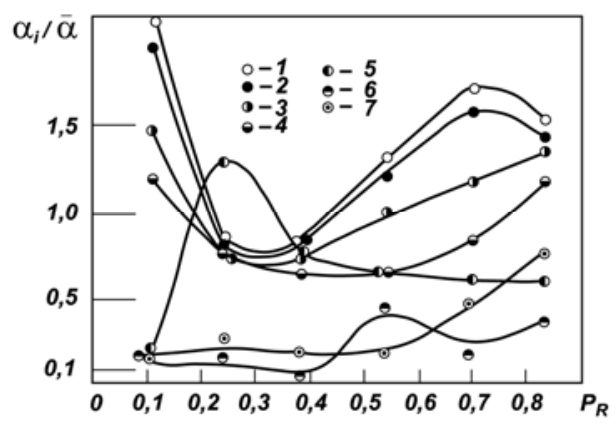

Fig. 6. Distribution of the relative heat transfer coefficient over the height of type 1 fin $(h / d=0.932)$ located in the first (along the gas flow) row. $\left.\left.\left.\operatorname{Re}=2 \cdot 10^{4} . \varphi, o: 1\right) 0 ; 2\right) 30 ; 3\right) 60$; 4) 90 ; 5) 120 ; 6) 150; 7) 180

The peaks of $a_{i} / a_{a v}$ below the middle section of the tube (curves for $\varphi>90^{\circ}$ ), observed in Fig. 6, stem from the spreading along the flow of the recirculation vortices from the fin root zones upon interaction with the layers that were sheared off from the supporting cylinder (tube). On the whole, the value of $a_{i} / a_{a v}$ in the rear part of the fin is lower because of formation (at high $h / d$ ) of a broad trailing vortex zone with relatively weak recirculation flow. For the same reason Fig. 6 does not exhibit any significant rise in $a$ on the rear side of the tube itself. The slight rise in $a$ on the rear edge of the fin at $\varphi=120$ to $180^{\circ}$ is produced by macroscale vortices, into which the sheared off layers (mentioned above) that separate from the tube are rolled up by the secondary flows. The large size of the rear vortex zone at high $h / d$ is due to the significantly greater thickness of the boundary layer on the tall fin and, as a result, significant displacement of flow from the interfin space.

At low relative fin heights $(h / d \leq 0.357)$, the flow pattern on the fin (Fig. $7 \mathrm{~b})$ and the distribution of $a_{i} / a_{a v}$ over its surface (Fig. 8) change. The effect of the tube itself on the flow pattern in the windward part of the fin increases, increasing the strength of the root vortex in region $A_{2}$ and forming of a local pressure trough beneath its center. As a result, an adverse pressure gradient is induced in the zone of the recirculating flow. This gradient causes separation of the boundary layer from the fin surface in zone $A_{3}$ and the formation, in addition to the small corner zone $A_{1}$ of still another circulation zone, namely $A_{3}$. The secondary flow is reattached in zone $A_{4}$ and then continues counter the main stream up to the windward edge of the fin. In this way the zone of secondary flow extends to the front 
edge of the fin. The net result is that much of the fin area is virtually devoid of flow patterns predicted by the traditional theory.

The distribution of $a_{i} / a_{a v}$ over the fin surface fits the above flow pattern. In the windward half of the fin, the largest $a_{i} / a_{a v}$ occurs in the root region. As in the case of $h / d=0.932$, this is caused by secondary flows. However, at the Re $=1 \cdot 10^{4}$ (Fig. 8a) there are two peaks in this region: the first is close to the base $\left(P_{R}<0.15\right)$ in the point of reattachment of the secondary flow to the fin surface, while the second is slightly farther from the base $\left(P_{R}=0.33\right)$. The second peak is induced by the transition from laminar to turbulent flow in the wall boundary layer in the accelerated flow region of zone $A_{2}$ (Fig. $7 \mathrm{~b}$ ). The second peak is not observed at higher Re (Fig. $8 \mathrm{~b}$ ) because the boundary-layer flow is now turbulent until the reattachment point. At all Re we observed beyond this point a reduction in $a_{i} / a_{a v}$, which dips in the point of separation of the secondary flow from the fin surface (zone $A_{3}$, Fig. $7 \mathrm{~b}$ ) at approximately midheight of the fin $\left(P_{R}=0.5\right)$.
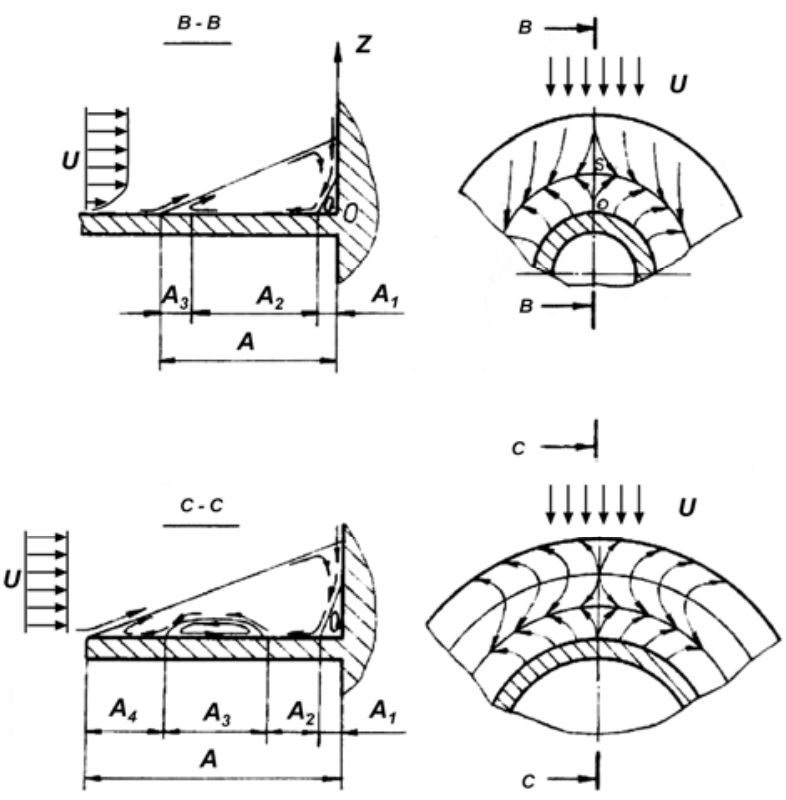

Fig. 7. Patterns of flow upstream of the windward part of the finned tube. a) At high $h / d ; b$ ) at low $h / d ; A$ zone of secondary circulation flows; $A_{1}$ and $A_{3}$ zones of separation of the boundary layer from the surface; $A_{2}$ and $A_{4}$ reattachment regions

The $a_{i} / a_{a v}$ in the rear part of the fin are higher than in the case of high $h / d$, mainly because of the more intensive recirculation flows in the relatively shallower and wider trailing vortical zone. These flows create three-dimensional secondary flows in the root zone of the rear part of the fin $\left(\varphi>120^{\circ}\right.$ and $\left.P_{R}<0.2\right)$, which are qualitatively similar to the recirculation flows in the front root zone $A$. Their superposition on the high flow turbulence in the rear one accounts for the high $a_{i} / a_{a v}$ in the rear of the tube at low $h / d$.

Note that at $h / d=0.263$ maximum $a$ occurs at the fin base at all Re. These results show up quite clearly in Figs. 9 and 10. 


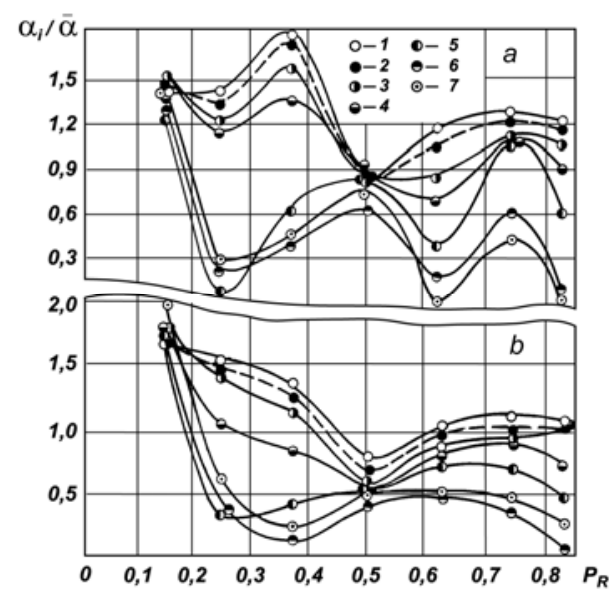

Fig. 8. Distribution of the relative heat transfer coefficient over the height of type 2 fin $(h / d=0.357)$ located in the first (in the direction of gas flow) row. Re: a) $\left.1 \cdot 10^{4} ; \mathrm{b}\right) 2 \cdot 10^{4} . \varphi, \mathrm{o}$ : 1) 0 ; 2) 30 ; 3) 60;4) 90; 5) 120; 6) 150; 7) 180

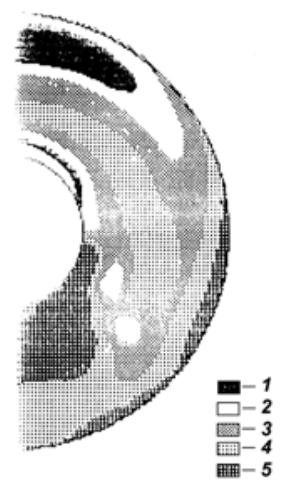

Fig. 9. Distribution of the relative heat transfer coefficient over the surface of type 1 fin $(h / d=$ 0.932 ) located in the frontal row of the bundle. $\operatorname{Re}=2 \cdot 10^{4}$; the free-stream direction is from the top down. $a_{i} / a_{\mathrm{av}}$ : 1) 1.93 to 1.53 ; 2) 1.53 to 1.14 ;3) 1.14 to 0.75 ; 4) 0.75 to 0.35 ; 5) 0.35 to 0.03

The above is also confirmed by the spike in turbulence level $\varepsilon$ on the rear surface of the tube, as well as by the fact reported in (Neal \& Hitchcock, 1966) that the variations in $\varepsilon$ in the zones of $\varphi=0$ and $180^{\circ}$ at low $h / d$ are similar. The local minima and maxima of the curves of $a_{i} / a_{a v}=f\left(P_{R}\right)$ at $\varphi>90$ o (Fig. 8a) are probably caused by transitions in the recirculation boundary layer in the rear region of the fin, and also by propagation of secondary flows from the frontal zone of $A$ toward the rear. With reduction in row pitch $\sigma_{2}$, the distribution of $a$ over the fin surfaces of the inner-row tubes is increasingly affected by the type of tube arrangement within the bundle (Figs. 11 and 12). Thus, the value of $a$ on the front parts $(\varphi \approx$ $\pm 30^{\circ}$ ) of fins of the inner tubes in in-line bundles decreases steeply along (Fig. 12a) because 
of the effect of vortical wake of the upstream tubes in the same rows (these values of $a$ drop to those typical of the rear vortical zones of the upstream tubes).

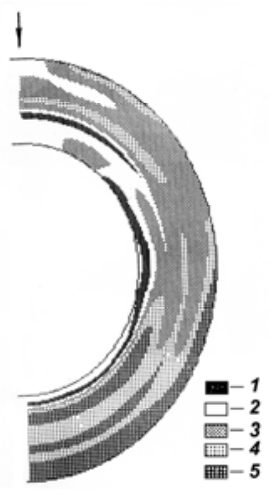

Fig. 10. Distribution of the relative heat transfer coefficient over the surface of type 2 fin $(h / d=0.357)$ located in the frontal row of the bundle. $R e=2 \cdot 10^{4}$; the free-stream direction is from the top down. $\left.a_{\mathrm{i}} / a_{\mathrm{av}}: 1\right) 1.94$ to 1.66 ; 2) 1.66 to 1.24 ; 3) 1.24 to 0.83 ; 4$) 0.83$ to 0.41 ;

5) 0.41 to 0.11

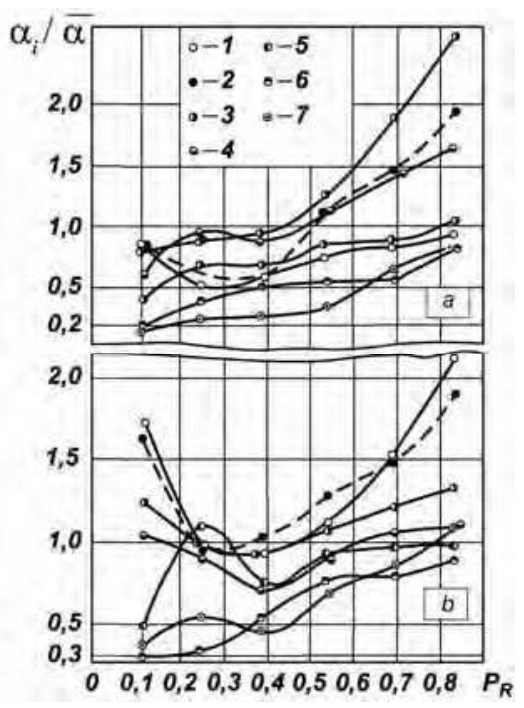

Fig. 11. Distribution of the relative heat transfer coefficient over the surface of type 1 fin $(h / d=0.932)$ in the 4 th row of a six-row bundle. $\operatorname{Re}=2 \cdot 10^{4}$; for legend see Fig. 7 or 9 .

a) in-line bundle, $\sigma_{1}=3.47, \sigma_{2}=2.97 ; \mathrm{b}$ ) staggered bundle, $\sigma_{1}=3.47, \sigma_{2}=2.66$

The drop in $a$ increases at smaller $\sigma_{2}$, and is explainable by the decrease of the intensity of circulation in the wake with decreasing relative pitch $L / d$ between interacting tubes (for inline bundles $L / d=\sigma_{2}$ ). According to data from (Migay, 1978), the rear wake exhibits an 
approximately constant turbulence level $\varepsilon$. Thus, in the inner rows of in-line bundles, the highest $a$ occur in fin zones with $\varphi \approx \pm 50$ to $70^{\circ}$, where the fin is impacted by a flow outside its aerodynamic shadow.

In a staggered bundle with longitudinal and transverse pitches similar in those in an in-line bundle, the $L / d$ is double that of the latter bundle $\left(L / d=2 \sigma_{2}\right)$, so that at $\sigma_{2}>2$ the distributions of $a$ on the fronts of inner-row tubes (Fig. 12b) stays approximately the same as on the first row, i.e., with a peak at $\varphi=0^{\circ}$. The uniformity of the distributions of $a$ in these bundles improves with the forcing effect of adjoining tubes, which is maximum at $\sigma_{1} / \sigma_{2}=$ $2 \sqrt{ } 3$. In this case each tube operates as if it were surrounded by a circular deflector formed of six adjoining tubes.

As $\sigma_{2}$ in staggered bundles is decreased to $\sigma_{2}<1.5$ (which is possible at quite large $\sigma_{1}$ and relatively low $h / d$ ), the flow pattern begins to resemble that in in-line bundles. That is, the inner-row tubes operate in the near vortex wakes of upstream tubes, and the distributions of $a$ over the fin circumference (Fig. 13) acquire the configuration exhibiting the low $a$ in the front that is typical of in-line bundles. It remains to represent the experimental data on the local values of $a$ in dimensionless form. In paper (Pis'mennyi, 1991), in which we described the surface-average values of $a$ for bundles of transversely finned tubes, the high values of exponent $\bar{m}$ in the equation for the average heat transfer coefficients

$$
N \bar{u}=C \operatorname{Re}^{\bar{m}}
$$

which are typical of bundles with low $L / d$, were attributed to a direct correlation between the values of $\varepsilon$ and $m$. Workup of data on local $a$ for a type 1 finned tube (Table 1 ) located in an inner row of an in-line bundle with $\sigma_{1}=3.47$ and $\sigma_{2}=2.97$ confirmed the existence of this correlation.
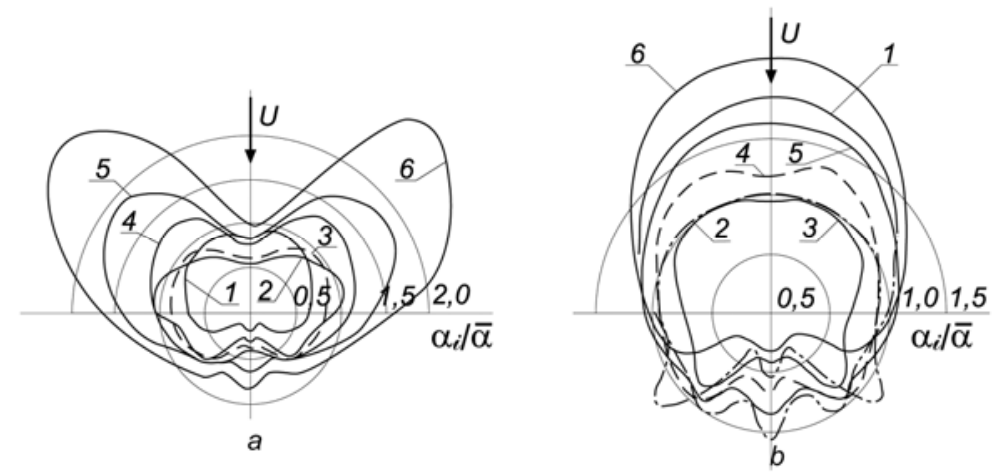

Fig. 12. Distribution of the relative heat transfer coefficient over the circumference of type 1 fin $(h / d=0.932)$ in the 4 th row of a six-row bundle. $R e=2.104$; a) in-line bundle, $\sigma_{1}=3.47$, $\sigma_{2}=2.97$; b) staggered bundle, $\sigma_{1}=3.47, \sigma_{2}=2.66 . P: 1$ ) 0.117 ; 2) 0.247 ; 3) 0.393; 4) 0.540; 5) 0.697 ; 6) 0.833

The highest levels of $\varepsilon$ in both the front and rear vortical wakes correlate with high values of $m$ in the equation for the local heat transfer coefficient

$$
\mathrm{Nu}_{l}=C_{l} \operatorname{Re}^{m}
$$


These results are listed in Table 3 in a form convenient for comparison with Figs. 11a and $12 \mathrm{a}$, which present the distributions of $a$ for this bundle. Averaging of local values over the surface of the finned tube yields $\bar{m}=0.836$, which is virtually identical to the value of $\bar{m}=0.833$, calculated from the correlation in (Pis'mennyi, 1993; Pis'mennyi \& Terekh, 1991).

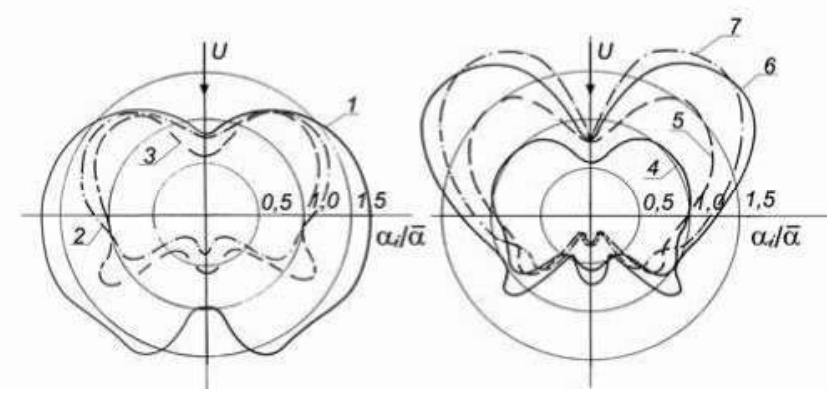

Fig. 13. Distribution of the relative heat transfer coefficient over the circumference of type 2 fin $(h / d=0.357)$ in the $5^{\text {th }}$ row of a seven-row bundle with $\sigma_{1}=3.33$ and $\sigma_{2}=1.30 . \operatorname{Re}=2 \cdot 10^{4}$. P: 1) 0.13 ; 2) 0.22 ; 3) 0.34 ; 4) 0.47 ; 5) 0.60 ; 6) 0.72 ; 7) 0.81

\begin{tabular}{|c|c|c|c|c|c|c|c|}
\hline \multirow{2}{*}{$\mathrm{P}$} & \multicolumn{7}{|c|}{ Local values of $\bar{m}$} \\
\cline { 2 - 8 } & $0^{\circ}$ & $30^{\circ}$ & $60^{\circ}$ & $90^{\circ}$ & $120^{\circ}$ & $150^{\circ}$ & $180^{\circ}$ \\
\hline 0.117 & 0.86 & 0.80 & 1.00 & 1.07 & 1.30 & 1.22 & 1.30 \\
\hline 0.247 & 0.88 & 0.82 & 0.80 & 0.77 & 0.88 & 0.85 & 1.04 \\
\hline 0.393 & 0.73 & 0.72 & 0.56 & 0.72 & 0.71 & 0.95 & 1.15 \\
\hline 0.540 & 0.77 & 0.65 & 0.58 & 0.64 & 0.82 & 0.99 & 1.10 \\
\hline 0.697 & 0.78 & 0.58 & 0.57 & 0.64 & 0.90 & 1.10 & 1.05 \\
\hline 0.893 & 0.91 & 0.78 & 0.62 & 0.69 & 0.94 & 1.06 & 0.89 \\
\hline
\end{tabular}

Table 3. Values of $\bar{m}$ in the equation for the local heat transfer coefficient

We have thus gained deeper insight into the physics of the processes occurring in bundles of transversely finned tubes, improved our understanding of the temperature distributions in standard industrial tube bundles operating at high heat flux densities.

The developed heat transfer surfaces applied in large power plants have, as a rule, a staggered arrangement with large lateral $S_{1}$ and small longitudinal $S_{2}$ tube pitches, for which there are corresponding increased values of the parameter $S_{1} / S_{2}=2.5$ to 4.0. Large values of $S_{1}$ are dictated by a need for ensuring repairs of the heat exchange device. Besides, the bundles with large lateral pitch are less contaminated and more fitted for cleaning. On the other hand, relatively small values of the longitudinal pitch $S_{2}$ are dictated by a need for providing sufficient compactness of the heat exchange device as a whole.

As results for the flow (Pis'mennyi, 1991) and local heat transfer revealed, the arrangement parameters $\left(S_{1}, S_{2}\right.$, and $\left.S_{1} / S_{2}\right)$ largely determine the flow past the bundles and the distribution of heat transfer rates over their surface.

Dimensions of the rear vortex zone are at a maximum in the bundles characterized by large values of parameter $S_{1} / S_{2}$. In such bundles, the neighboring tubes exert a slight reducing effect on the flow in interfin channels and, being displaced as the boundary layer at the fin 
thickens in the direction from the axis of the incident flow, the flow forms a wide rear zone (Fig. 14).

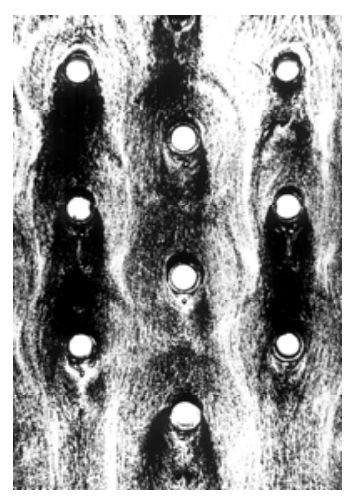

Fig. 14. Flow pattern in the finned tube bundle with $S_{1} / S_{2}=3.0\left(\operatorname{Re}=5.3 \cdot 10^{4}\right)$ (Pis'mennyi, 1991)

In this case, the distribution of heat transfer rates over the finned tube surface is essentially uneven: in the forepart of a circular diagram of the relative heat transfer coefficients there is a crevasse associated with a superposition of the near vortex wake from the streamwise preceding tube (Fig. 13). The same pattern is observed also in the rear part of the tube. Thus, frontal and rear sections of the finned tubes, which are in the region of aerodynamic shadow in the discussed cases of large values of parameter $S_{1} / S_{2}$, show low-efficiency. In this case, the highest levels of the heat transfer rate are displaced into the lateral regions of tubes interacting with the flow outside the zone of the aerodynamic shadow.

In the typical case considered there are two ways of increasing thermoaerodynamic efficiency of the heat transfer surface:

- the first way is linked with constructive measures that make it possible to engage lowefficient sections of the finned tube surface in a high-rate heat transfer; and

- the second way involves the use of heat transfer surfaces not having a finned part that lies in the region of aerodynamic shadow and is, in fact, useless.

\section{Bundles of the tubes with the fins bent to induce flow convergence}

The first of the two ways is applied to the case of finned tubes with circular cross section. It is suggested that this be done by bending the fins to induce flow convergence (Fig. 15).

This method of a development of the idea of parallel bending of fins suggested at the Podol'sk Machine Building Plant (Russian Federation) (Ovchar et al., 1995) in order to reduce the transverse pitches of tubes in bundles and to improve the compactness of heat exchangers as a whole. Surfaces with fins bent to induce flow convergence can be made of ordinary tubes with welded or rolled on transverse fins, by deforming the latter, something that is achieved by passing the finned tube through a "draw plate" or another kind of bending device. In addition to parameters of bundles of ordinary finned tubes, the geometry of such surfaces is described by two additional quantities: the convergence angle $\gamma$ and bending ratio $\mathrm{b} / \mathrm{h}$. For this reason the possibility of attaining the maximum enhancement of heat transfer when using the suggested method for tubes with specified values of $d, h, t$, and 
$\delta$, in addition to finding their optimal layout represented by ratios $\sigma_{1}$ and $\sigma_{2}$, involves finding the optimal values of $\gamma$ and $b / h$. Special investigations were performed for determining the extent of the enhancement and the optimum values of the above parameters.
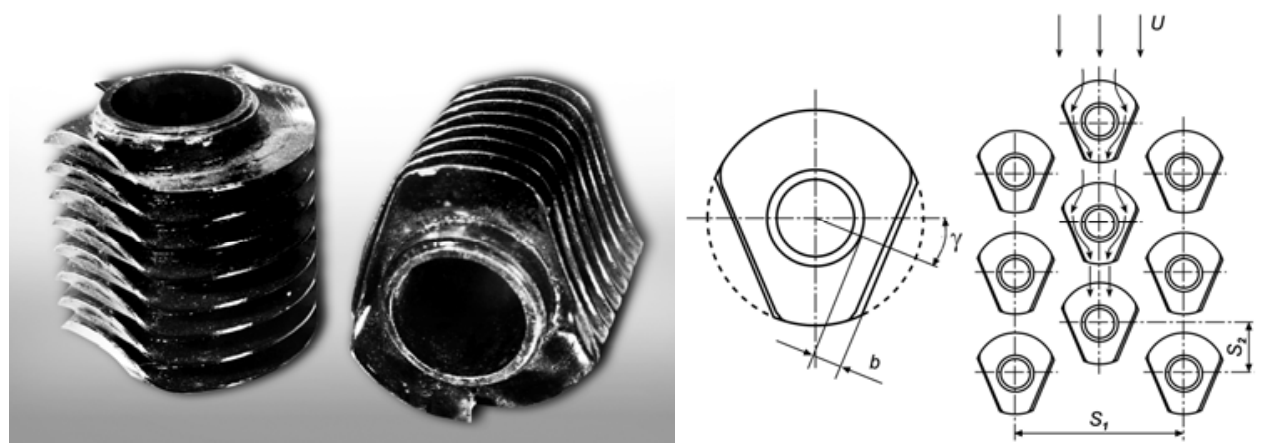

Fig. 15. Tubes with the fins bent to induce flow convergence

Studies of heat transfer, aerodynamic drag and specifics of flow over bundles of tubes with fins to bend in order to induce convergent flow were carried out using experimental methods, the most important features of which are:

- complete thermal simulation attained by electrically heating all the tubes in the bundle;

- determination, in the course of experiments, of surface-average convective heat transfer coefficients, by measuring the temperature distribution over the surface of the fin and of the wall of the finned tube.

The experiments were performed using steel tubes with welded-on transverse fins and the following geometric parameters: $d=42 \mathrm{~mm}, h=15 \mathrm{~mm}, t=8 \mathrm{~mm}, \delta=1.3 \mathrm{~mm}, \psi=5.98$, and $b / h=0.5$. Tubes with these dimensions are extensively used in various heat exchangers, including units used in power equipment.

The effect of the value of $\gamma$ on the thermoaerodynamic performance of finned-tube bundles was determined with the specially constructed bundles with $\gamma=7^{\circ}, 14^{\circ}$, and $20^{\circ}$.

The value of $b / h$ was selected with consideration of investigations of heat transfer and aerodynamic drag of the bundles of tubes with parallel bent fins, which showed that the value of $b / h$ for tubes of these dimensions should be taken equal to 0.5 . A further increase in this ratio causes a marked rise in drag while contributing virtually nothing to heat transfer enhancement.

Calorimetering tubes that served for measuring the temperature field of the fin and the tube were made of turned steel blanks in the form of two parts screwed together with one another. This provided access to the surface of the tube heightwise middle fin into which, as into the wall of the tube at its base, were lead-caulked in 18 copper-constantan thermocouples that used $0.1 \mathrm{~mm}$ diameter wires. The beads of the latter were, prior to this, welded in points with specified coordinates. The thermocouples were installed at a pre-bent fin. The fins were bent by pressing the tube in a specially constructed "draw plate" with a specified distance and angle between bending plains. The device was capable of producing fins with different values of $\gamma$.

The geometric parameters of the staggered tube bundles used in the experiments are listed in Table 4. 


\begin{tabular}{|c|c|c|c|c|c|c|}
\hline $\begin{array}{c}\text { Location } \\
\text { number }\end{array}$ & $S_{1}, \mathrm{~mm}$ & $S_{2}, \mathrm{~mm}$ & $\sigma_{1}$ & $\sigma_{2}$ & $\sigma_{1} / \sigma_{2}$ & $d_{\text {eq }}, \mathrm{mm}$ \\
\hline 1 & 135 & 38 & 3.21 & 0.90 & 3.55 & 26.9 \\
\hline 2 & 135 & 54 & 3.21 & 1.29 & 2.50 & 34.6 \\
\hline 3 & 135 & 65 & 3.21 & 1.55 & 2.08 & 38.3 \\
\hline 4 & 135 & 75 & 3.21 & 1.79 & 1.80 & 38.3 \\
\hline 5 & 135 & 85 & 3.21 & 2.02 & 1.59 & 38.3 \\
\hline 6 & 127 & 38 & 3.02 & 0.90 & 3.34 & 23.8 \\
\hline 7 & 111 & 54 & 2.64 & 1.29 & 2.06 & 27.0 \\
\hline 8 & 86 & 75 & 2.05 & 1.79 & 1.15 & 17.1 \\
\hline 9 & 86 & 85 & 2.05 & 2.02 & 1.01 & 17.1 \\
\hline
\end{tabular}

Table 4. Geometric parameters of the bundles of tubes with fins bent to induce flow convergence

A total of 24 staggered tube bundles were used in the experiments; the planes of the bent parts of the fins of all the tubes were oriented symmetrically relative to the direction of the free stream. The surface-average heat transfer of internal rows of tubes was investigated at Re between $3 \cdot 10^{3}$ and $6 \cdot 10^{4}$. The experimental data were approximated by power-law equations in the form

$$
\mathrm{Nu}=\mathrm{C}_{\mathrm{q}} \cdot \operatorname{Re}^{\mathrm{m}} \text {. }
$$

Table 5 lists value of experimental constants $m$ and $C_{q}$ in equation (5) for the 24 bundles that were investigated. The extent of heat transfer enhancement was assessed by comparing our data with those for ordinary bundles (in which the fins were not bent).

Analysis of results shows that bending the fins enhances heat transfer in all the cases under study, but that its level, defined by the ratio of Nusselt numbers for the experimental and basic fins $\left(\mathrm{Nu} / \mathrm{Nu}_{b}\right)$, depends highly on the value of $\gamma$ and on the tube pitches (Fig. 16).

As expected, the highest values of $\mathrm{Nu} / \mathrm{Nu}_{b}$ were obtained in bundles with large transverse and relatively small longitudinal pitches $\left(\sigma_{1} / \sigma_{2}>2\right)$ when the conditions of washing the leading and trailing parts of basic finned tubes are highly unfavorable (Pis'mennyi, 1991).

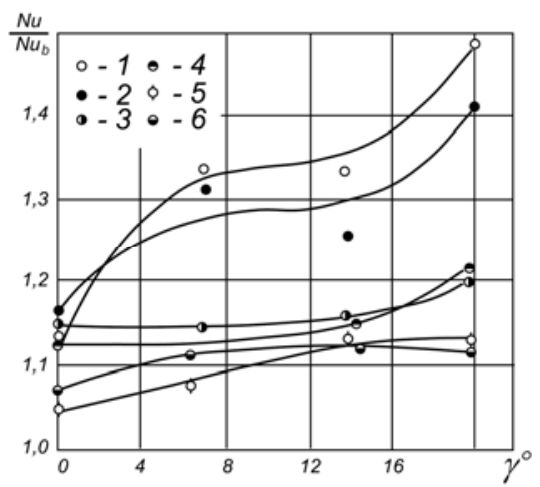

Fig. 16. Enhancement of heat transfer as a function of convergence angle $\gamma$ at $\operatorname{Re}=1.3 \cdot 10^{4}$. $\sigma_{1}=3.21$; $\left.\sigma_{2}: 1\right) 1.29$; 2) 1.55 ; 3) 1.79 ; 4) 2.02; $\sigma_{1}=2.05$; $\left.\sigma_{2}: 5\right) 1.79$; 6$) 2.02$ 


\begin{tabular}{|c|c|c|c|c|c|c|c|c|}
\hline \multirow{2}{*}{$\begin{array}{c}\text { Location } \\
\text { number }\end{array}$} & \multirow{2}{*}{$\sigma_{1}$} & \multirow{2}{*}{$\sigma_{2}$} & \multicolumn{2}{|c|}{$r=7^{\circ}$} & \multicolumn{2}{c|}{$r=14^{\circ}$} & \multicolumn{2}{c|}{$r=20^{\circ}$} \\
\cline { 4 - 9 } & & $m$ & $C_{\mathrm{q}}$ & $m$ & $C_{\mathrm{q}}$ & $m$ & $C_{\mathrm{q}}$ \\
\hline 1 & 3.21 & 0.90 & - & - & 0.69 & 0.135 & 0.71 & 0.112 \\
\hline 2 & 3.21 & 1.29 & 0.64 & 0.270 & 0.66 & 0.232 & 0.71 & 0.158 \\
\hline 3 & 3.21 & 1.55 & 0.67 & 0.204 & 0.70 & 0.150 & 0.68 & 0.196 \\
\hline 4 & 3.21 & 1.79 & 0.66 & 0.187 & 0.69 & 0.151 & 0.68 & 0.162 \\
\hline 5 & 3.21 & 2.02 & 0.61 & 0.276 & 0.69 & 0.148 & 0.67 & 0.185 \\
\hline 6 & 3.02 & 0.90 & - & - & 0.77 & 0.068 & 0.74 & 0.084 \\
\hline 7 & 2.64 & 1.29 & - & - & 0.73 & 0.132 & 0.76 & 0.111 \\
\hline 8 & 2.05 & 1.79 & 0.71 & 0.105 & 0.71 & 0.107 & 0.71 & 0.107 \\
\hline 9 & 2.05 & 2.02 & 0.66 & 0.155 & 0.71 & 0.102 & 0.72 & 0.094 \\
\hline
\end{tabular}

Table 5. Experimental constants $m$ and $C_{\mathrm{q}}$ in Eq. (5)

The bent tube segments in this case press the flow toward the trailing part of the finned tube, thus directing highly-intense secondary flows that are generated in the root region of the leading part of the tube (Pis'mennyi, 1984; Pis'mennyi \& Terekh, 1993b) deeper into the space downstream of the tube. This, in the final analysis, decreases markedly the size of the trailing vertical zone, which is clearly seen by comparing Figs. 17a and b, obtained by visualizing the flow on the standard and bent fins of tubes of the same dimensions under otherwise same flow conditions. Significant segments of the trailing surfaces of the tube and fin then participate in high-rate heat transfer, thus increasing the overall surface-average heat transfer rate. This rate increases both because of reduction in the size of regions with low local velocities and by increasing the fraction of the surface of the finned tube that interacts with high-intensity secondary circulating flows, which are induced to come into contact with the peripheral lateral parts of the fin and also due to increasing the length of vortex filaments within a given area (Fig. 18).

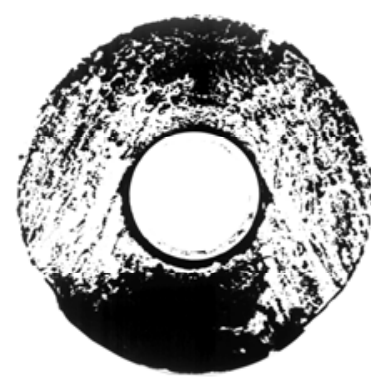

a

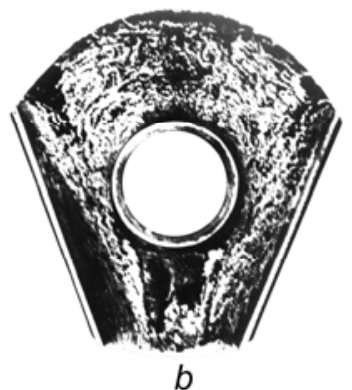

b

Fig. 17. Flow on the surface of an ordinary cylindrical (a) and bent (b) fins at $\operatorname{Re}=2 \cdot 10^{4}$

The flow pattern in the wake of the finned tube also changes radically. The leading part of the further downstream tube interacts in this case with a relatively intensive jet that is discharged from the trailing convergent part of the tube-fins set (Fig. 15), rather than with the ordinarily encountered weak recirculation flow. This also increases the heat transfer coefficient, because of the increase in the local velocities and also because of intensification of secondary circulation flows at the fin root and increasing the region of their activity in the leading part of the finned tube (Fig. 18). 

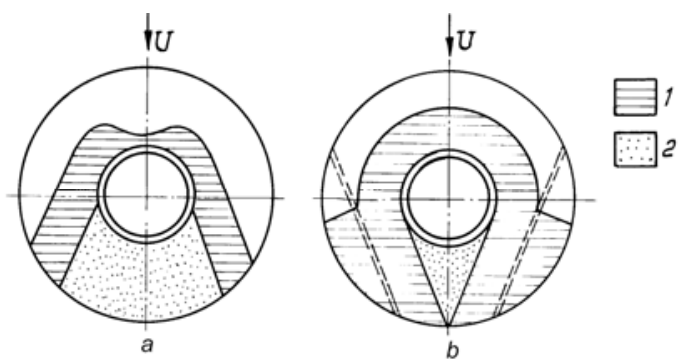

Fig. 18. Transformation of the dimensions of typical regions on the surface of a finned tube in the inward part of a bundle with $\sigma_{1} / \sigma_{2}>2$ with fin bent to provide for flow convergence. (a) an ordinary (basic) fin, and (b) bent fin. 1) region of intensive secondary circulating flows; 2) the trailing vortex zone

The level of perturbation of the wake flow which, as is known, controls, together with the local velocities, the rate of heat transfer remains rather high with the bent fins. This is promoted by turbulization of the flow after its separation from the outer surfaces of the perforated wall of the convergent "nozzle" that is formed by the bent parts of the fins (Fig. 15) and injection through gaps between their edges of a part of the flow from the spaces between the fins transverse to the free stream (Fig. 19).
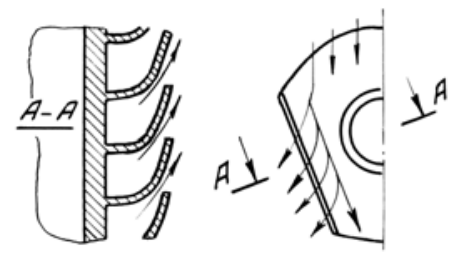

Fig. 19. Injection of flow into the space between the tubes through slots in the walls of the "convergent nozzle"

Taken together, all the above increases the surface-averaged heat transfer coefficient. Here exist optimal values of $\sigma_{2}$ which give, in case of $\sigma_{1} / \sigma_{2}>2$ under study, the greatest gain in the heat transfer coefficient. Thus, at $\sigma_{1}=3.21$ the value of $\mathrm{Nu} / \mathrm{Nu}_{b}$ is highest at $\sigma_{2} \approx 1.3$. The slight deterioration in the improvement at lower values of $\sigma_{2}$ is caused by increasing the mutual shading of tubes of the deeper-lying rows, which interferes with the supply of "fresh" flow from the spaces between the tubes to the convergent passages formed by the bent fins. A much greater reduction in the value of $\mathrm{Nu} / \mathrm{Nu}_{b}$ is observed when the value of $\sigma_{2}$ is increased above the optimal. This is also caused by redistribution of the flow in the spaces between the tubes and the fins so as to reduce the flow rates within the latter.

The dominant effect of the relationship between the flow rates in the spaces between the fins and those between the tubes is also confirmed by the fact that reducing the values of $\sigma_{1}$ while maintaining the values of $\sigma_{1} / \sigma_{2}$ constant causes blockage of spaces between the tubes, over which a part of the flow was bypassed past the convergent passages formed by the fins (Fig. 20), which causes the flow rate through the latter to increase.

It is typical that the maximum gain in the rate of heat transfer is observed in layouts that also provide for the highest absolute values of the surface-average heat transfer coefficients. 

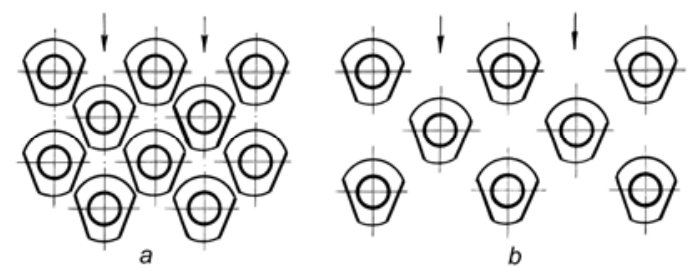

Fig. 20. Comparison of configurations of bundles with $\sigma_{1}=3.21, \sigma_{2}=1.55\left(\sigma_{1} / \sigma_{2}=2.08\right)$ (b) and with $\sigma_{1}=2.64, \sigma_{2}=1.29\left(\sigma_{1} / \sigma_{2}=2.06\right)(\mathrm{a})$

As previously mentioned, the effect of $\gamma$ on the rate of heat transfer is very clearly observed, but is much more complex than it would appear at first sight. This is seen from Fig. 16 which, in addition to data obtained in the present experimental study at $\gamma$ between 7 and $20^{\circ}$, also presents experimental results on bundles of the same size with parallel bending of fins $\left(\gamma=0^{\circ}\right)$. The effect of $\gamma$ is most perceptible at the ranges between 0 to $7^{\circ}$ and 14 to $20^{\circ}$. As noted, the effect of the fin bending ratio $b / h$ on the heat transfer rate was investigated using tubes with parallel fin bending. Experiments performed over the range of $b / h=0.3$ to 0.5 showed that $\mathrm{Nu} / \mathrm{Nu}_{b}$ increases only slightly (up to $5 \%$ ) with an increase in $b / h$. There are grounds to believe that this tendency prevails also when the fins are bent to provide flow convergence.

It was found in investigating the aerodynamic drag of bundles of tubes with flowconvergence inducing bending of fins that the experimental data at $\operatorname{Re}_{e q}$ between $3 \cdot 10^{3}$ and $6 \cdot 10^{4}$ are satisfactorily approximated by an expression such as

$$
\mathrm{Eu}_{0}=C_{r} \cdot \operatorname{Re}_{e q}{ }^{-n} \text {. }
$$

Table 6 lists the values of experimental constants $n$ and $C_{r}$ for the tube bundles under study. Bending of fins to provide for flow convergence was found to cause a marked rise in the aerodynamic drag as compared with bundles where the fins were not so bent over the entire range of pitches, pitch ratios and values of $\gamma$. The rise in drag can be represented by the ratio of Euler number for the bundle under study and for the base bundle $\mathrm{Eu}_{0} / \mathrm{Eu}_{0}{ }^{b}$ at $\operatorname{Re}_{e q}=$ const.

It is seen from Fig. 21 that the variation in $\mathrm{Eu}_{0} / \mathrm{Eu}_{0} b=f(\gamma)$ is monotonous. The highest rise in drag (to $90-100 \%$ ) is observed at $\gamma=20$. These data were compared with separately obtained results for tubes with parallel bent fins. It is remarkable that the rise in $\mathrm{Eu}_{0} / \mathrm{Eu}_{0} b$ as compared with the case of $\gamma=0^{\circ}$ does not exceed $30 \%$. This indicates that inducing convergence of flow in the spaces between the fins is only one of the reasons of the rise in drag in such bundles. Another factor is that bending of fins as such, even at $\gamma=0$, causes a transformation of the half-open spaces between the fins into narrow closed curved channels with wedge-shape cross sections (Fig. 19), the flow between which involves a marked energy loss, in particular because it is subjected to the decelerating effect of the walls over the entire perimeter of its cross section.

It follows from the analysis above that improving the flow pattern within the bundle may allow attaining a significant rise in the heat transfer rate without an excessive increase in drag. Depending on the fin-bending parameters, layout and Reynolds number for the tubes of the size under study the enhancement of heat transfer ranges from 15 to $77 \%$ at a respective rise in drag between 40 and $11 \%$ as compared with ordinary fins. 


\begin{tabular}{|c|c|c|c|c|c|c|c|c|}
\hline \multirow{2}{*}{$\begin{array}{c}\text { Location } \\
\text { number }\end{array}$} & \multirow{2}{*}{$\sigma_{1}$} & \multirow{2}{*}{$\sigma_{2}$} & \multicolumn{2}{|c|}{$\gamma=7^{\circ}$} & \multicolumn{2}{c|}{$\gamma=14^{\circ}$} & \multicolumn{2}{c|}{$r=20^{\circ}$} \\
\cline { 4 - 9 } & & $n$ & $C_{\mathrm{r}}$ & $n$ & $C_{\mathrm{r}}$ & $n$ & $C_{\mathrm{r}}$ \\
\hline 1 & 3.21 & 0.90 & - & - & 0.11 & 0.744 & 0.15 & 1.271 \\
\hline 2 & 3.21 & 1.29 & 0.15 & 1.410 & 0.15 & 1.473 & 0.16 & 1.775 \\
\hline 3 & 3.21 & 1.55 & 0.14 & 1.319 & 0.17 & 1.717 & 0.17 & 1.857 \\
\hline 4 & 3.21 & 1.79 & 0.13 & 0.943 & 0.15 & 1.280 & 0.16 & 1.485 \\
\hline 5 & 3.21 & 2.02 & 0.13 & 0.859 & 0.14 & 1.080 & 0.17 & 1.615 \\
\hline 6 & 3.02 & 0.90 & - & - & 0.13 & 1.070 & 0.14 & 1.263 \\
\hline 7 & 2.64 & 1.29 & - & - & 0.14 & 1.626 & 0.13 & 1.553 \\
\hline 8 & 2.05 & 1.79 & 0.13 & 1.065 & 0.14 & 1.256 & 0.13 & 1.176 \\
\hline 9 & 2.05 & 2.02 & 0.13 & 1.073 & 0.14 & 1.173 & 0.14 & 1.241 \\
\hline
\end{tabular}

Table 6. Experimental constants $n$ and $C_{r}$ in Eq. (6)

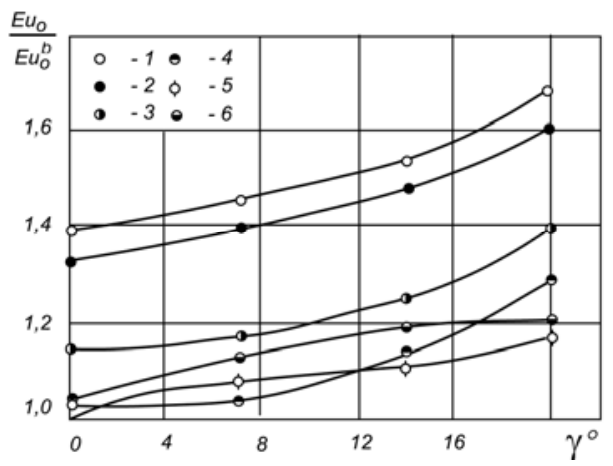

Fig. 21. Rise in aerodynamic drag as a function of $\gamma$ at $\left.\operatorname{Re}=1.3 \cdot 10^{4} . \sigma_{1}=3.21 ; \sigma_{2}: 1\right)=1.29$; 2) 1.55 ; 3) 1.79 ; 4) 2.02 ; $\sigma_{1}=2.05$; $\sigma_{2}$ : 5) 1.79 ; 6) 2.02

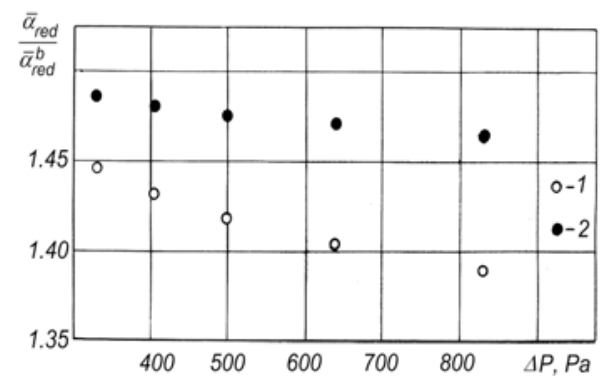

Fig. 22. Ratio of surface-averaged reduced heat transfer coefficients of the enhanced and basis bundles at the same values of drag and $\sigma_{2}=1.29$; $\left.\sigma_{1}: 1\right) 3.21$;) 2.64

The effect of using a given method of enhancement of external heat transfer in finned-tube bundles can be uniquely estimated by comparing the reduced heat transfer coefficients of the ordinary and enhanced bundles at equal pressure drops $\Delta P$. Estimates performed in this 
manner show that the best performance is exhibited by bundles with $\sigma_{2}=1.29$ and $\sigma_{1}=3.21$ and 2.64 at $\gamma=20$. Figure 22 is a plot of the ratio of surface-averaged reduced heat transfer coefficients $\bar{\alpha}_{\text {red }}$ of enhanced and basis bundles obtained at $\Delta P=$ idem.

The range of values of drag corresponds to Re between $8 \cdot 10^{3}$ and $13 \cdot 10^{3}$ which is most typical for power-equipment heat exchangers. It follows from the figure that the net gain in the external heat transfer of convergence-inducing bending of fins for $\sigma_{1}=3.21$ and $\sigma_{2}=1.29$ is from 38 to $44 \%$ and for the case of $\sigma_{1}=264$ and $\sigma_{2}=1.29$ it is at least $47 \%$. Metal consumption of the device decreases correspondingly.

\section{Surfaces of partially finned flattened oval tubes}

The second of the ways for improving the thermoaerodynamic performance of transverselyfinned heat transfer surfaces that involves removing ineffective parts of fins appears advisable in cases when configured (oval, flattened-oval, etc.) finned tubes are used in heat exchangers in order to reduce the aerodynamic drag. In such cases it is suggested to replace fully finned configured (for example, flattened-oval) tubes by partially finned ones, i.e., such in which parts of the cylindrical surface with a high curvature (the leading and trailing parts) are not finned (Fig. 23). This means that the suggested type of surface is missing a part of the fin area which "works" relatively poorly not only because it, as a rule, is located in the region of the aerodynamic shadow, but also because its efficiency factor $E$ is lower than that for fins located on the flat lateral sides of the tube.

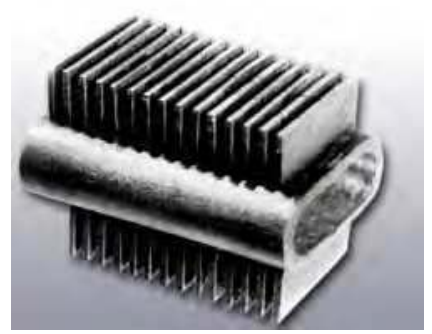

Fig. 23. Partially finned flattened oval tubes

The principal geometric parameters of the tubes (Table 7) were selected to be close to those of fully finned oval tubes, the heat transfer and aerodynamics of which were investigated in (Yudin \& Fedorovich, 1992). This made it possible to compare their thermoaerodynamic performance and to evaluate the effect of replacing fully finned tubes by those with a partially finned surface.

The surface-average heat transfer was investigated by the traditional method of complete thermal modeling consisting in electric heating of all the tube bundles. The main quantity of interest were the reduced heat transfer coefficients $a_{\text {red. }}$. The heat transfer coefficients $a$ were computed from the reduced coefficients using the expression

$$
\mathrm{a}_{\mathrm{red}}=\mathrm{a}\left(\mathrm{E} \cdot \mathrm{H}_{\mathrm{f}} / \mathrm{H}_{\mathrm{t}}+\mathrm{H}_{\mathrm{ft}} / \mathrm{H}_{\mathrm{t}}\right)
$$

The fin efficiency factor $E$ was calculated from a formula for a straight rectangular fin. For comparison of the heat transfer data with corresponding data for fully-finned tubes from the paper (Yudin \& Fedorovich, 1992), which also presents reduced heat transfer coefficients, 
the latter were also recalculated to their convective counterparts by means of equation (7). The values of $E$ for the oval fin were then determined by averaging values calculated separately for segments with smaller and greater curvature over the surface.

It is sensible to compare heat transfer data for tubes with different fin patterns only when the convective heat transfer coefficients are referred to the surface of the tube, for which reason the experimental results were represented in the form

$$
\mathrm{Nu}_{\mathrm{c}} \cdot \psi=f(\operatorname{Re})
$$

\begin{tabular}{|l|c|c|c|}
\hline Quantity & Designation & $\begin{array}{l}\text { Partially } \\
\text { finned tubes }\end{array}$ & $\begin{array}{l}\text { Fully finned } \\
\text { tubes } 1\end{array}$ \\
\hline Transverse dimension of finned tube & $d_{1}, \mathrm{~mm}$ & 15.0 & 14.0 \\
\hline Longitudinal dimension of finned tube & $d_{2}, \mathrm{~mm}$ & 38.0 & 36.0 \\
\hline Height of fins & $h, \mathrm{~mm}$ & 11.5 & 10.0 \\
\hline Fin pitch & $t, \mathrm{~mm}$ & 3.5 & 3.0 \\
\hline Fin thickness & $\delta, \mathrm{mm}$ & 0.8 & 0.5 \\
\hline Surface extension factor & $\Psi$ & 5.22 & 10.2 \\
\hline Aspect ratio of tube cross section & $d_{2} / d_{1}$ & 2.53 & 2.57 \\
\hline Relative height of fin & $h / d_{1}$ & 0.76 & 0.71 \\
\hline Relative fin pitch & $t / d_{1}$ & 0.23 & 0.21 \\
\hline Relative fin thickness & $\delta / t_{1}$ & 0.05 & 0.04 \\
\hline
\end{tabular}

Table 7. Geometric parameters of configured finned tubes

The data on aerodynamic drag were represented in the form of Euler numbers referred to a single transverse row of a bundle.

The experiments were performed with seven staggered and two in-line bundles, in which the flattened oval tubes were arranged with their major axis along the free-stream velocity vector (Fig. 24a and d).

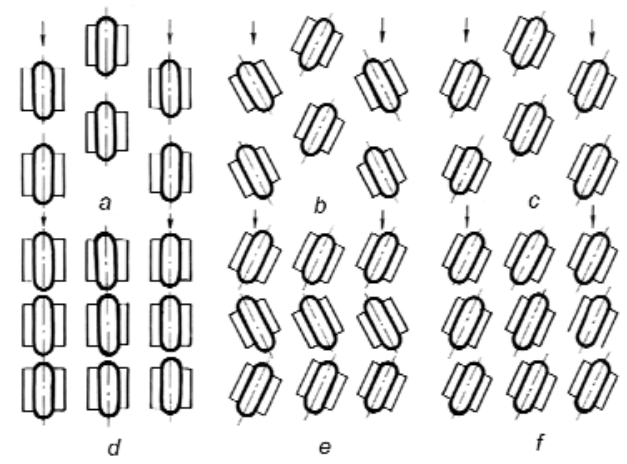

Fig. 24. Geometric arrangements of configured tubes within the bundles: (a) through (c) staggered bundles; (d) through (f) in-line bundles

1 Yudin \& Fedorovich, 1992 
At the same time, configured tubes can be placed within bundles in a number of ways by varying the angle of attack of their profile $\Theta$, and also by using different combinations of mutual arrangement of the tubes with nonzero angle $\Theta$. In this manner we analyzed two principal versions of in-line and staggered arrangements: with successive alternation of the sign of angle $\Theta$ across the bundle (Fig. 24b and e) and without such alternation (Fig. 24c and f). This means that we investigated a total of 15 versions of bundle arrangements. Their geometric parameters are listed in Table 8.

The results on heat transfer (Fig. 25) show, in the first place, that replacing fully finned oval tubes $(\psi=10.2)$ with partially finned tubes $(\psi=5.22)$ does not reduce the heat flux from the tube bundle, all other conditions remaining equal. This holds for all the four bundle geometries (Nos. 1, 2, 4, and 5, Table 8) that had pitches which allowed comparison with data for bundles of fully finned tubes obtained in (Yudin \& Fedorovich, 1992), which validates the physical assumptions for the modification of the tubes. Moreover, the heat flux removed from bundles of partially finned tubes is in these cases even slightly higher than from bundles of fully finned tubes. The mutual location of curves of $\mathrm{Nu}_{\mathrm{k}} \cdot \psi=f(\operatorname{Re})$ for all pairs of bundles being compared (curves for tubes with $\psi=10.2$ lie lower and are shallower) allows the assumption that the reason for the lower heat transfer efficiency of the fully finned tubes is the existence of thermal contact resistance between the oval fins that have been placed on them and the tube wall, the role of which increases with increasing $R e$, as follows from paper (Kuntysh, 1993). On the other hand, partially finned tubes have a perfect thermal contact between the fins and the tube wall.

\begin{tabular}{|c|c|c|c|c|c|c|c|c|}
\hline $\begin{array}{c}\text { Arrange } \\
\text {-ment } \\
\text { number }\end{array}$ & $\begin{array}{c}\text { Bundle } \\
\text { geometry }\end{array}$ & $\mathrm{S}_{1}, \mathrm{~mm}$ & $\begin{array}{c}\mathrm{S}_{2,} \\
\mathrm{~mm}\end{array}$ & $\mathrm{~S}_{1} / d_{1}$ & $\mathrm{~S}_{2} / d_{1}$ & $\mathrm{~S}_{1} / \mathrm{S}_{2}$ & $\Theta$ o & $\begin{array}{c}\text { Bundle } \\
\text { geometry }\end{array}$ \\
\hline 1 & staggered & 47.5 & 46.0 & 3.17 & 3.07 & 1.03 & 0 & $\mathrm{a}$ \\
\hline 2 & $"$ & 47.5 & 58.0 & 3.17 & 3.87 & 0.82 & 0 & $\mathrm{a}$ \\
\hline 3 & $"$ & 47.5 & 75.0 & 3.17 & 5.00 & 0.63 & 0 & $\mathrm{a}$ \\
\hline 4 & $"$ & 63.3 & 36.0 & 4.22 & 2.40 & 1.73 & 0 & $\mathrm{a}$ \\
\hline 5 & $"$ & 63.3 & 42.0 & 4.22 & 2.80 & 1.51 & 0 & $\mathrm{a}$ \\
\hline 6 & $"$ & 63.3 & 46.0 & 4.22 & 3.07 & 1.38 & 0 & $\mathrm{a}$ \\
\hline 7 & $"$ & 63.3 & 58.0 & 4.22 & 3.87 & 1.09 & 0 & $\mathrm{a}$ \\
\hline 8 & $"$ & 63.3 & 46.0 & 4.22 & 3.07 & 1.38 & 30 & $\mathrm{~b}$ \\
\hline 9 & $"$ & 63.3 & 46.0 & 4.22 & 3.07 & 1.38 & 30 & $\mathrm{c}$ \\
\hline 10 & in-line & 63.3 & 46.0 & 4.22 & 3.07 & 1.38 & 0 & $\mathrm{~d}$ \\
\hline 11 & $"$ & 63.3 & 58.0 & 4.22 & 3.87 & 1.09 & 0 & $\mathrm{~d}$ \\
\hline 12 & $"$ & 63.3 & 46.0 & 4.22 & 3.07 & 1.38 & 15 & $\mathrm{e}$ \\
\hline 13 & $"$ & 63.3 & 46.0 & 4.22 & 3.07 & 1.38 & 30 & $\mathrm{e}$ \\
\hline 14 & $"$ & 63.3 & 46.0 & 4.22 & 3.07 & 1.38 & 15 & $\mathrm{f}$ \\
\hline 15 & $"$ & 63.3 & 46.0 & 4.22 & 3.07 & 1.38 & 30 & $\mathrm{f}$ \\
\hline
\end{tabular}

Table 8. Geometric parameters of the bundles of configured partially finned tubes

Investigations of the effect of bundle configuration showed that at the same pitches and Reynolds numbers in-line bundles have virtually one half of the drag of staggered bundles.

2 as depicted in Fig. 24 
The in-line geometry gives on the average 40 to $50 \%$ lower values of $a$ as compared with the staggered bundle with the same values of $S_{1}$ and $S_{2}$, or which reason the effect of pitch at $\Theta=0^{\circ}$ was investigated primarily with staggered bundles.

The heat transfer coefficient varied by 20 to $25 \%$ over the range of $S_{1} / d_{1}$ between 3.17 and 4.22, of $S_{2} / d_{1}$ from 2.4 to 5 and $S_{1} / S_{2}$ between 1.03 and 1.76: it increased with $S_{1} / S_{2}$ and with $S_{1} / d_{1}$ and decreased and stabilized with increasing $S_{2} / d_{1}$. The highest heat transfer coefficients were obtained with arrangement $4\left(S_{1} / d_{1}=4.22\right.$ and $\left.S_{2} / d_{1}=2.4\right)$.

This allows the assumption that in certain cases in-line bundles of configured finned tubes may become preferable to staggered bundles.

The effect of $S_{1} / d_{1}$ and $S_{2} / d_{1}$ on the drag was investigated in staggered bundles. The data show that for the given pitches of bundles of partially finned tubes decreases with an increase in both these geometric ratios. As to the effect of angle of attack $\Theta$, it was found (Fig. 26) that the drag increases markedly with increasing $\Theta$ both in staggered and in-line bundles. The drag is virtually independent on the mutual arrangements of the tubes at $\Theta \neq 0$ (Fig. 24). Still it appears that $\Theta$ has a somewhat more perceptible effect on the drag of in-line as compared with staggered bundles: in the first case increasing $\Theta$ from $0^{\circ}$ to $30^{\circ}$ at $\operatorname{Re}_{f_{s}}=10^{4}$ increases $\mathrm{Eu}_{0}$ by approximately $90 \%$, whereas in the second - by approximately $70 \%$. In addition, the shape of curves of $\mathrm{Eu}_{0}=f\left(\operatorname{Re}_{f s}\right)$ for the in-line bundles changes with $\Theta$ : in the case of $\Theta-30^{\circ}$ the curves become virtually self-similar $(n \approx 0)$ over the entire range of $\operatorname{Re}$ under study, whereas at $\Theta=0$ they have a perceptible slope $(n=-0.16)$. On the other hand, for staggered bundles these curves are virtually equidistant both at $\Theta=0^{\circ}$ and $30^{\circ}$.

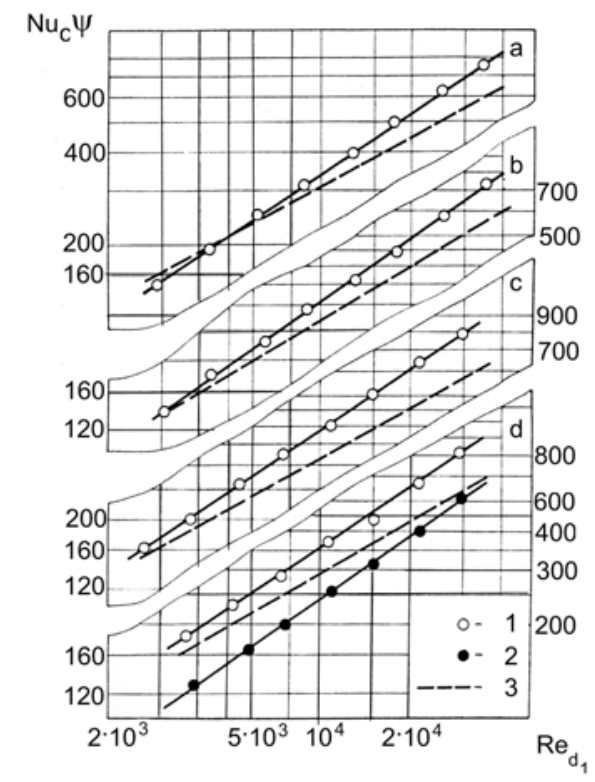

Fig. 25. Heat transfer from bundles of configured finned tubes at $\Theta=0$. a) $S_{1} / d_{1}=3.17$, $S_{2} / d_{1}=3 / 07$; b) $S_{1} / d_{1}=3.17, S_{2} / d_{1}=3.87$; c) $S_{1} / d_{1}=4.22, S_{2} / d_{1}=2.40 ;$ d) $S_{1} / d_{1}=4.22$, $\left.S_{2} / d_{1}=2.80 ; 1\right)$ staggered bundles of partially finned tubes; 2$)$ in-line bundle of partially finned tubes; 3) staggered bundles of fully finned tubes (Yudin \& Fedorovich, 1992) 


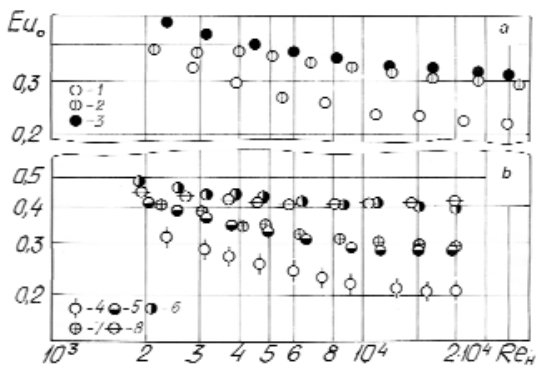

Fig. 26. Aerodynamic drag of bundles of partially finned tubes at $\Theta>0$. a) staggered bundles; b) in-line bundles; 1) geometry $6 a ; 2)$ geometry $8 b ; 3)$ geometry $8 c ; 4$ ) geometry $10 d$; 5) geometry $12 \mathrm{e}$; 6) geometry $13 \mathrm{e}$; 7 ) geometry $14 \mathrm{f} ; 8$ ) geometry $15 \mathrm{f}$ (the geometry numbers correspond to Table 8)

\section{Conclusions}

Thus, the use of tubes with fins bent to induce flow convergence makes it possible to markedly reduce the weight and size of heat exchangers under the same thermal effectiveness. In addition, the suggested type of enhanced finned surfaces is of interest also in the following aspects:

- tubes with fins of the suggested type can be manufactured employing standard technologies of rolling-on and welding-on of fins coupled with a relatively simple finbending operation, i.e., does not require extensive retooling and large additional expenditures; and

- bundles equipped with fins of the suggested type should exhibit better self-cleaning properties in dust laden flows than bundles using standard finned tube, since foulants usually accumulate in the aerodynamic shadow zone in the rear and front parts of the finned tube.

It is possible to use bundles of partially finned configured tubes which, in the first place, will allow a large saving of fin metal. On the assumption that heat fluxes removed from two bundles with similarly spaced fully and partially finned tubes with the same heights $h$, pitches $t$, fin thicknesses $\delta$, shape and dimensions of the tubes are at least equal, then, if their aerodynamic drag values are also equal, replacing these by the others may save about half of the metal used for fins of fully finned tubes. This may amount to $20-30 \%$ of the total weight of the heat exchanger. The reasons why the heat flux density removed from these two types of tubes remains the same and maybe even increases somewhat in spite of the reduction in the heat-transmission area may be the following:

- the fins that are eliminated are parasitic, since they are usually located in the aerodynamic shadow;

- the fins placed on the flat lateral surfaces of flattened oval and similar tubes have efficiency $E$ higher than oval fins;

- the technology of producing partially finned tubes allows providing for virtually ideal thermal contact between the fins ant the tube wall, which is not true of the currently employed technologies of producing fully finned oval tubes; and

- the elimination of the leading and trailing parts of the fins eliminates additional thermal resistance in the form of foulants that deposit between these fins. 


\section{Nomenclature}

$d$ - diameter of finned tube;

$d_{1}$ and $d_{2}$ - lateral and longitudinal dimensions of the cross section of the shaped tube, respectively;

$E$ - fin efficiency factor;

$h$ - fin height;

$H$ - heat-transfer area;

$P_{R}$ - dimensionless coordinate: $\left(r-r_{0}\right) /\left(R-r_{0}\right)$;

$r_{0}$ and $R$ - radius of finned tube at the basis and end of the fin, respectively;

$S_{1}$ and $S_{2}$ - transverse and longitudinal tube pitches, respectively;

$t$ - fin pitch;

$\Delta P$ - pressure drop;

$U$ - flow velocity;

$\Psi$ - fin factor;

$\Theta$ - inclination angle of a logitudinal axis of the shaped tube cross section to the velocity vector of the incident flow;

$\sigma_{1}=S_{1} / d$ and $\sigma_{2}=S_{2} / d$ - relative transverse and longitudinal tube pitches, respectively;

$\delta$ - fin thickness

Subscripts:

eq - equivalent;

$f_{s}$ - free stream;

$f t$ - surface of non-finned part of the tube;

red-reduced

\section{References}

Tolubinskiy, V.I. \& Lyogkiy, V.M. (1964). Heat Transfer Coefficients and Aerodynamic Drags of Single Finned Cylinders in Cross Air Flow (in Russian). Voprosy Radioelektroniki, Ser.1, Electronika, Issue 9, pp. 114-120

Migai, V.K., Bystrov, P.G., \& Fedotov, V.V. (1992). Heat Transfer in the Bundles of Tubes with Lug-Type Finning in Cross Flow (in Russian). Heavy Mechanical Engineering (Тяжелое машиностроение), No.7, pp. 8-10

Eckels, P.W. \& Rabas, T.J. (1985). Heat Transfer and Pressure Drop Performance of Finned Tube Bundles. Journal of Heat Transfer, Vol.107, pp. 205-213

Taranyan, I.G., Iokhvedov, F.M., \& Kuntysh V.B. (1972). Study of the Effect of Finning Parameters on Heat Transfer and Drag of Staggered Bundles of Tubes with Transverse Smooth and Integral Fins (in Russian). Thermophysics of High Temperatures (Теплофизика высоких температур), Vol.10, No.5, pp. 1049-1054

Kuntysh, V.B. \& Iokhvedov, F.M. (1968). Heat Transfer and Aerodynamic Drag of the Bundles of Tubes with Slotted Fins (in Russian). Refrigerating Engineering (Холодильная техника), No.6, pp. 14-18

Antufiev, V.M., \& Gusyev, Ye.K. (1968). Enhancement of Heat Transfer of Finned Surfaces in Cross Flow (in Russian). Heat Power Engineering (Теплоэнергетика), No.7, pp. 31-34

Iokhvedov, F.M., Taranyan, I.G., \& Kuntysh, V.B. (1975). Heat Transfer and Aerodynamic Drag of Staggered Tube Bundles with Various Shapes of a Transverse Slotted Fin (in Russian). Power Mechanical Engineering (Энергомашиностроение), No.11, pp. 23-26

Sparrow, E.M., \& Myrum, T.A. (1985). Crossflow Heat Transfer for Tubes with Periodically Interrupted Annular Fins. International Heat Mass Transfer, Vol.28, No.2, pp. 509-512 
Weierman, C. (1976). Correlations to Ease the Selection of Finned Tubes. Oil and Gas Journal, Vol.74, No.36, pp. 94-100

Antufiev, V.M. (1965). Study of Efficiency of Various Shapes of Finned Surfaces in Cross Flow (in Russian). Heat Power Engineering, No.1, pp. 81-86

Kokorev, V.I., Vishnevskiy, V.U., Semyonov, S.M. et al. (1978). Results of Studying Heat Transfer Tubes with Slotted Transverse Fins (in Russian). Heat Power Engineering, No.2, pp. 35-37

Kuntysh, V.B. \& Piir, A.E. (1991). Enhancement of Heat Transfer of the Tube Bundles of Air Cooling Devices by Notching the Edges of Spiral Rolled-on Fins (in Russian). Izvestiya VUZov. Energetika, No.8, pp. 111-115

Kuntysh, V.B. (1993). Enhancement of Heat Transfer of Staggered Tube Bundles by Peripheral Notching of Spiral Fins (in Russian). Izvestiya VUZov. Energetika, No.5-6, pp. 111-117

Fiebig, M., Mitra, N., \& Dong, Y. (1990). Simultaneous Heat Transfer Enhancement and Flow Loss Reduction of Fin-Tubes. Heat Transfer-1990. Proceedings of 9th International conference, Vol.4, pp. 51-55, 1990, (Jerusalem, August 19-24), New York

Kuntysh, V.B. \& Kuznetsov, N.M. (1992). Thermal and Aerodynamic Calculations of Finned AirCooled Heat Exchangers (in Russian). Energoatomizdat Press, St. Petersburg Division, 280 p.

Yevenko, V.I. \& Anisin, A.K. (1976). Improving the Efficiency of Heat Transfer of the Tube Bundles in Cross Flow (in Russian). Heat Power Engineering (Теплоэнергетика), No.7, pp. $37-40$

Lokshin, V.A., Fomina V.N., \& Titova Ye.Ya. (1982). On One of the Methods of Enhancing Convective Heat Transfer in Smooth Tube Bundles in Cross Flow (in Russian). Heat Power Engineering (Теплоэнергетика), No.11, pp. 17-18

Migai, V.K. \& Firsova, E.V. (1986). Heat Transfer and Drag of Tube Bundles (in Russian). Nauka Press, 195 p.

Kuntysh, V.B., Stenin, N.N., \& Krasnoshchyokin, L.F. (1991). Study of Thermoaerodynamic Characteristics of Staggered Bundles with Nontraditional Arrangement of Finned Tubes (in Russian). Refrigerating Engineering (Холодильная техника), No.6, pp. 11-13

Kuntysh, V.B. \& Stenin, N.N. (1993). Heat Transfer and Aerodynamic Drag of In-line Staggered Finned Tube Bundles in Cross Flow (in Russian). Heat Power Engineering (Теплоэнергетика), No.2, pp. 41-45

Stenin, N.N. (1994). The Development and Study of Promising Arrangements of Finned Tubes for Air-Cooled Heat Exchangers (in Russian). Abstract of Candidate's Dissertation. St. Petersburg, $21 \mathrm{p}$.

Kuntysh, V.B., Piir, A.E., \& Gerasimenko A.N. (1990). Heat Transfer and Aerodynamic Drag of Staggered Bundles with a Variable Number of Tubes in a Row (in Russian). Izvestiya VUZov, Energetika, No.5, pp. 82-86

Pis'mennyi, E.N. (1991). Special Features of Flow and Heat Transfer in Staggered Bundles of Transversely Finned Tubes. Journal of Engineering Physics, Vol.60, No.6, pp. 676-681

Kuntysh, V.B. \& Fedotova, L.M. (1983). Effect of the Attack Angle of the Air Flow on Heat Transfer and Drag of a Staggered Finned Tube Bundle (in Russian). Izvestiya VUZov, Energetika, No.4, pp. 93-96

Samie, F. Sparrow, E. (1986). Heat Transfer from a Finned Tube Oriented at an Angle to Flow. Heat Transfer, No.2, pp. 205-208

Khavin, A.A. (1989). The Effect of the Angle of Incidence of Flow on Thermoaerodynamic Characteristics of Finned Tube Bundles (in Russian). Institute of Engineering Thermophysics (Институт технической теплофизики АН УССР), Dep. In VINITI, No.6957-V89

Antufiev, V.M. (1966). Efficiency of Various Shapes of Convective Heating Surfaces (in Russian). Energiya Press, 184 p. 
Berman, Ya.A. (1965). Study and Comparison of Finned Tubular Heat Transfer Surfaces in a Wide Range of Reynolds Numbers (in Russian). Chemical and Oil Mechanical Engineеring (Химическое и нефртяное машиностроение), No.10, pp. 21-26

Yudin, V.F. \& Fedorovich, Ye.D. (1992). Heat Transfer of Oval-Shaped Finned Tube Bundles (in Russian). The International Minsk Forum 1992, Convective Heat Transfer, Minsk, Vol.1, Part 1, pp. 58-61

Ilgarubis, V.-A.S., Ulinskas, R.B., \& Butkus, A.V. (1987). Drag and Average Heat Transfer of Compact Plane-Oval Finned Tube Bundles (in Russian). Proceedings of the Academy of Sciences of Lithuanian SSR (Trudy Akad. Nauk LitSSR), Set B, Vol.158, pp. 49-55

Ota, T., Nishiyama, H., \& Taoka, Y. (1984). Heat Transfer and Flow around an Elliptic Cylinder. International Journal of Heat Mass Transfer, Vol.27, No.10, pp. 1771-1776

Pis'mennyi, E.N. \& Lyogkiy, V.M. (1984). Toward the Calculation of Heat Transfer of Multi Row Staggered Bundles of Tubes with Transverse Finning. Thermal Engineering, No.31 (6), pp. 349-352

Pis'mennyi, E.N. (1984). Study of Flow on the Surface of Fins on Cross Finned Tubes. Journal of Engineering Physics, Vol.47, No.1, pp. 761-765

Pis'mennyi, E.N. \& Terekh, A.M. (1993a). A Generalized Method for Calculating Convective Heat Transfer with Cross Flow over Tube Banks Having External Annular and Coil-Tape Finning. Thermal Engineering, Vol. 40, No.5, pp. 394-398

Pis'mennyi, E.N. \& Terekh, A.M. (1993b). Local Heat Transfer in Bundles of Transversely Finned Tubes. Heat Transfer Research, Vol.25, No.6, pp. 825-835

Skrinska, A.J., Žukauskas, A.A., \& Štašiulevičius, J.K. (1964). An Experimental Study of the Local Coefficients of Heat Transfer from Helically-Finned Tubes (in Russian). Proceedings of the Academy of Sciences of Lithuanian SSR (Trudy Akad. Nauk LitSSR), Set B, Vol.4 (39), pp. 213-218

Žukauskas, A.A., Ulinskas, R.V., \& Zinevičius, F.V. (1984). Local Parameters of Heat Transfer and Flow over Bundles of Staggered Finned Tubes (in Russian). Ibid., Vol.2 (141), pp. 46-55

Neal, S.B.H.C. \& Hitchcock, J.A. (1966). A Study of the Heat Transfer Processes in Bundles of Finned Tubes in Cross Flow Using a Large Model Technique. Proceedings of the $3^{\text {rd }}$ International Heat Transfer Conference (Chicago), pp. 290-293

Lyogkiy, B.M., Zholudov, Ya.S., \& Gerashchenko, O.A. (1976). Local Heat Transfer in Crossflow over a Single Circular Tube with External Circular Fins (in Russian). Journal of Engineering Physics (Инженерно-физический журнал), Vol.30, No.2, pp. 274-280

Krückels, W. \& Kottke, V. (1970). Untersuchung über die Verteilung des Wärmeübergangs an Rippen und Rippenrohr-Modellen. Chemie-Ing. Technik, Bd. 42, No.6, S. 355-362

Gardon, R. (1960). A Transducer for the Measurement of Heat Flow Rate. Journal of Heat Transfer, Vol.82, No.4, pp. 396-398

Migay, V.K. (1978). Calculation of Heat Transfer in Bundles of Staggered Tubes Operating in Crossflow (in Russian). Heat Power Engineering (Теплоэнергетика), No.2, pp. 31-34

Pis'mennyi, E.N. \& Terekh, A.M. (1991). Heat Transfer in Bundles of Transversely Finned Tubes with Small Numbers of Tube Rows (in Russian). Industrial Heat Engineering (Промышиенная теплотехника), Vol.13, No.3, pp. 55-60

Ovchar, V.G. et al. (1995). Certain Aspects of Improving the Performance of Steam Boilers and Thermal Electric Power Plants (in Russian). Heat Power Engineering (Теплоэнергетика), No.8, pp. 2-8

Kuntysh, V.B. (1993). Investigation of Heat Transfer and Its Enhancement in Tube Bundles of Air-Cooled Heat Exchangers (in Russian). Abstract of Doctor's Dissertation. St. Petersburg, 45 p. 


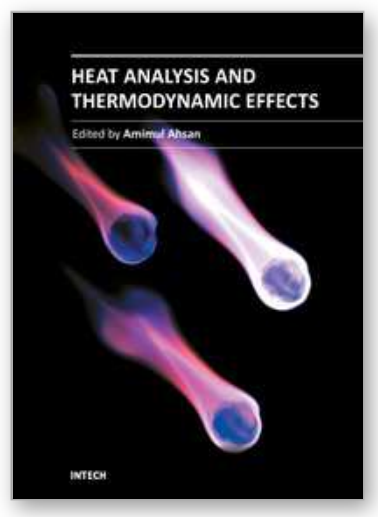

\author{
Heat Analysis and Thermodynamic Effects \\ Edited by Dr. Amimul Ahsan
}

ISBN 978-953-307-585-3

Hard cover, 394 pages

Publisher InTech

Published online 22, September, 2011

Published in print edition September, 2011

The heat transfer and analysis on heat pipe and exchanger, and thermal stress are significant issues in a design of wide range of industrial processes and devices. This book includes 17 advanced and revised contributions, and it covers mainly (1) thermodynamic effects and thermal stress, (2) heat pipe and exchanger, (3) gas flow and oxidation, and (4) heat analysis. The first section introduces spontaneous heat flow, thermodynamic effect of groundwater, stress on vertical cylindrical vessel, transient temperature fields, principles of thermoelectric conversion, and transformer performances. The second section covers thermosyphon heat pipe, shell and tube heat exchangers, heat transfer in bundles of transversely-finned tubes, fired heaters for petroleum refineries, and heat exchangers of irreversible power cycles. The third section includes gas flow over a cylinder, gas-solid flow applications, oxidation exposure, effects of buoyancy, and application of energy and thermal performance index on energy efficiency. The forth section presents integral transform and green function methods, micro capillary pumped loop, influence of polyisobutylene additions, synthesis of novel materials, and materials for electromagnetic launchers. The advanced ideas and information described here will be fruitful for the readers to find a sustainable solution in an industrialized society.

\title{
How to reference
}

In order to correctly reference this scholarly work, feel free to copy and paste the following:

E.N. Pis'mennyi, A.M. Terekh and V.G. Razumovskiy (2011). Enhancement of Heat Transfer in the Bundles of Transversely-Finned Tubes, Heat Analysis and Thermodynamic Effects, Dr. Amimul Ahsan (Ed.), ISBN: 978953-307-585-3, InTech, Available from: http://www.intechopen.com/books/heat-analysis-and-thermodynamiceffects/enhancement-of-heat-transfer-in-the-bundles-of-transversely-finned-tubes

\section{INTECH}

open science | open minds

\author{
InTech Europe \\ University Campus STeP Ri \\ Slavka Krautzeka 83/A \\ 51000 Rijeka, Croatia \\ Phone: +385 (51) 770447 \\ Fax: +385 (51) 686166 \\ www.intechopen.com
}

\author{
InTech China \\ Unit 405, Office Block, Hotel Equatorial Shanghai \\ No.65, Yan An Road (West), Shanghai, 200040, China \\ 中国上海市延安西路65号上海国际贵都大饭店办公楼 405 单元 \\ Phone: +86-21-62489820 \\ Fax: +86-21-62489821
}


(C) 2011 The Author(s). Licensee IntechOpen. This chapter is distributed under the terms of the Creative Commons Attribution-NonCommercialShareAlike-3.0 License, which permits use, distribution and reproduction for non-commercial purposes, provided the original is properly cited and derivative works building on this content are distributed under the same license. 